\title{
Le musée de Sculpture comparée au prisme de la collection de cartes postales éditées par les frères Neurdein (1904-1915)
}

The Musée de Sculpture Comparée through the collection of postcards published by the Neurdein brothers [1904-15]

\section{Dominique Jarrassé et Emmanuelle Polack}

\section{(2) OpenEdition}

\section{Journals}

Édition électronique

URL : http://journals.openedition.org/cel/476

DOI : $10.4000 / \mathrm{cel} .476$

ISSN : 2262-208X

Éditeur

École du Louvre

Édition imprimée

Date de publication : 1 avril 2014

Référence électronique

Dominique Jarrassé et Emmanuelle Polack, « Le musée de Sculpture comparée au prisme de la collection de cartes postales éditées par les frères Neurdein (1904-1915) », Les Cahiers de l'École du Louvre [En ligne], 4 | 2014, mis en ligne le 01 avril 2014, consulté le 02 mai 2019. URL : http:// journals.openedition.org/cel/476 ; DOI : 10.4000/cel.476

es Cahiers de l'École du Louvre sont mis à disposition selon les termes de la licence Creative Commons Attribution - Pas d'Utilisation Commerciale - Pas de Modification 4.0 International. 


\title{
Cahiers de l'École du Louvre
} recherches en histoire de l'art, histoire des civilisations archéologie, anthropologie et muséologie

\section{Numéro 4. Avril 2014}

\author{
Le musée de Sculpture comparée au prisme \\ de la collection de cartes postales \\ éditées par les frères Neurdein (1904-1915) \\ Dominique Jarrassé et Emmanuelle Polack
}

Article disponible en ligne à l'adresse :

http://www.ecoledulouvre/cahiers-de-I'ecole-du-louvre/numero4avril2014/Jarrasse_Polack.pdf

Pour citer cet article :

Dominique Jarrassé et Emmanuelle Polack, « Le musée de Sculpture comparée au prisme de la collection de cartes postales éditées par les frères Neurdein (19041915) », Cahiers de l'École du Louvre, recherches en histoire de l'art, histoire des civilisations, archéologie, anthropologie et muséologie [en ligne] $n^{\circ} 4$, avril 2014, p. 2 à 20.

\section{cc) creative \\ B.: $\ominus \$$}

(C) École du Louvre

Cet article est mis à disposition selon les termes de la Licence Creative Commons Attribution - Pas d'utilisation commerciale - Pas de modification 3.0 non transposé. 


\section{Cahiers de l'École du Louvre recherches en histoire de l'art, histoire des civilisations archéologie, anthropologie et muséologie \\ Numéro 4. Avril 2014}

Sommaire

Éditorial

p. 1

\section{Étude}

Le musée de Sculpture comparée au prisme de la collection de cartes postales éditées par les frères Neurdein (1904-1915)

Dominique Jarrassé et Emmanuelle Polack

p. $2-20$

Dossier:

Les modalités de la collecte : rapt, troc, marché, fouilles, don...

Et leur impact sur l'objet

Dans collection, il y a collecte...

Introduction de Dominique Jarrassé.

p. $21-23$

Entrer en collection. Pour une ethnographie des gestes et des techniques de collecte

Julien Bondaz

p. $24-32$

De la meilleure façon de constituer une collection. Le cas des émaux «byzantins » de Mikhaill Botkine

Aglaé Achechova.

Edme Antoine Durand (1768-1835) : un bâtisseur de collections

Louise Detrez p. $45-55$

Réunir une documentation pour l'Art Brut : les prospections de Dubuffet dans l'immédiat après-guerre au regard du modèle ethnographique Baptiste Brun p. $56-66$

Travailler sur des objets en faisant se confronter les points de vue : regards croisés sur le langage fou : l'exemple des écrits bruts

Vincent Capt p. $67-75$

D’un regard-pilote à l'écart. L'impact de Dubuffet sur les collectes de l'association L'Aracine

Déborah Couette. p. $76-86$

La cabane éclatée. Morcellement des objets immobiliers apparentés à l'art brut

Roberta Trapani .p. $87-95$ 
Dominique Jarrassé et Emmanuelle Polack

Prendre des centaines et des centaines de clichés, en faire 1600 cartes postales éditées pour le "grand public ", mais aussi pour les savants, révèle un projet étonnant où se conjuguent les aspirations à l'exhaustivité qui caractérisent souvent archéologues et historiens - dont la prolifération des bases de données actuellement offre une indéniable résurgence -, le souci de fournir une documentation qui dépasse le seul souvenir d'une visite au musée et les usages de la photo dans le travail de l'historien, ce que l'on se plait à qualifier comme son atelier ${ }^{1}$. Ici il est même envisageable qu'une fonction de gestion ait été pensée par le conservateur qui présida à la réalisation par la maison Neurdein de cette immense série de cartes postales, Camille Enlart (1862-1927), lui-même photographe prolifique ${ }^{2}$. Sa boulimie de photos n'a d'égal que son goût pour la saturation des espaces des galeries du Trocadéro. Sa direction se caractérisa par la multiplication des moulages (on passe de 1297 en 1900 à 2147 en 1925) $)^{3}$ en lien avec une réorganisation des salles qu'il lance en 1903, au moment où, justement, la carte postale se répand intensément ${ }^{4}$. Cette conjonction n'est pas fortuite.

La carte postale, photo aux qualités inégales, produit de consommation courante, dévalorisé par sa prolifération même, mais immédiatement objet de collection et rapidement omniprésent dans les ateliers des artistes et les cabinets des historiens, a longtemps attendu une révision de son statut d'auxiliaire transparent en histoire de l'art, hormis le cas des cartes postales conçues d'emblée par le milieu artistique du type des Sturm-Postkarten ou de celles des Wiener Werstätte. Nous sommes indéniablement dans une phase de patrimonialisation ${ }^{5}$ et sa valeur marchande grandit, au gré de la conscience qui apparaît nettement qu'elle est irremplaçable, parfois témoin unique de certaines œuvres ou monuments ${ }^{6}$; elle entre pleinement dans le champ archivistique et historiographique, comme dans celui des études qui se multiplient sur l'" image-document ${ }^{7}$ " ou les dites Digital Humanities qui accompagnent ces mises en banque de données. D’ailleurs, la série de cartes postales du musée de Sculpture comparée a fait l'objet d'une numérisation

1. L'équipe de recherche de l'École du Louvre a mis sur pied un programme de recherche intitulé L'atelier de l'histoire de l'art. De la collecte aux humanités numériques qui s'attache à la mutation de la qualification des objets et à la fonction du « document » dans l'écriture de I'histoire de I'art. Cet article s'inscrit dans cette perspective de revalorisation d'un objet dont le statut est en pleine évolution : ainsi, en octobre 2011, I'Institut national du Patrimoine a proposé une formation intitulée La carte postale : source et patrimoine ; I'UMR Chastel porte un programme de recherche, Carte postale et création. Usages, fonctions, enjeux de la carte postale dans le champ artistique (XIXe-XXI ${ }^{e}$ siècles) qui a donné lieu à des colloques à Besançon en mai et à Paris en novembre 2013.

2. Plusieurs de ses livres sont illustrés de ses propres photos; toutefois il utilise aussi abondamment les photos des frères Neurdein, par exemple dans ses monographies du Musée (1911) ou de Rouen (1921). Ses photos sont conservées à la Médiathèque de l'Architecture et du Patrimoine et aux Archives de Boulogne-sur-Mer dans un ensemble de 18000 épreuves, dont les deux-tiers pris par Enlart, les autres étant de Neurdein, Braun...

3. Les auteurs remercient Christine Lancestremère, conservateur de la Galerie des Moulages au Musée des Monuments français, pour ces informations.

4. Il convient de signaler un article confus d'Axel Gampp, "Plaster Casts and Postcards: the postcard edition of the Musée de Sculpture Comparée, at Paris", Plaster Casts, Collecting and Displaying from Classical Antiquity to the Present, De Gruyter, pp. 501-517.

5. Parmi les programmes majeurs en ce domaine, l'exploitation de la collection Debuisson dans le cadre du projet du Musée d'Orsay/INHA, À nos grands hommes. La sculpture publique en France jusqu'à la Seconde Guerre mondiale (2004).

6. Processus conscient dès les restaurations du XIXe siècle et qui se traduit à travers les cartes : ex. la légende de la carte $\mathrm{n}^{\circ} 1100$, «Divinité asiatique » gallo-romaine, précise : « Original détruit lors du bombardement de Strasbourg ». Intéressante anticipation des fameuses étiquettes vertes qu'Enlart placera dans le musée pour signaler les œuvres détruites par les « barbares » germains, surtout après le bombardement de Reims.

7. Par exemple la série de rencontres Photo Archives and the Photographic Memory of Art History en 2009-2011 et la publication dirigée par Costanza Caraffa, Photo Archives and the Photographic Memory of Art History, Berlin, Deutscher Kunstverlag et parue en mai 2011. 
systématique ou quasiment, la rendant désormais accessible sur Internet ${ }^{8}$. Elle appartient à cette culture visuelle qui a trouvé un objet majeur de fascination dans l'Atlas Mnemosyne d'Aby Warburg. Nous l'envisagerons ici dans son double aspect d'objet photographique de diffusion, comme collection dont il convient de reconstituer l'histoire, et d'instrument d'une histoire de l'art à situer entre le catalogue encyclopédique et l'approche combinatoire d'unités indépendantes, fragmentaires... Car une série de 1600 cartes postales ne saurait relever du seul souci de rentabilité commerciale, elle ouvre sur une série d'usages plus complexes. Une partie de cette collection a plus de cent ans et reproduit des objets dont une partie a disparu. Par un jeu de reproductions en chaîne, elles témoignent de moulages qui eux-mêmes transmettent des états des monuments qui n’existent plus tels quels. Dans l'esprit des contemporains, les distances s'étaient quelque peu estompées - ainsi l'on envoyait de Vézelay ou de Chartres des cartes représentant des moulages!-, processus favorisé par le fait qu’à aucun moment il n'est précisé sur ces cartes postales que ce sont des photographies présentant des moulages et non les originaux'. C'est là une question centrale, celle du statut de ces cartes, reproductions de clichés photographiques montrant des moulages, eux-mêmes estampages de sculptures originales. Ici nous nous limiterons à comprendre la première partie de cette collection et la vision de l'histoire qu'elle propose.

Au parcours qui s'établit à travers les galeries du musée se superposent deux strates de photos, les 486 premières de la série suivent scrupuleusement la chronologie et la démonstration d'une évolution de l'antiquité à Rude et intègre encore quelques vues des moulages de sculptures khmères, tandis que les 1100 suivantes, tirées sans doute dans la continuité de la première collection, comme l'atteste l'usage de cartes dès 1906, rompent la logique pour tourner à une sorte d'inventaire à l'ordonnance duquel ne semble avoir présidé qu'un souci d'exhaustivité ${ }^{10}$, la succession dans la série perdant sa pertinence. Comment penser, au vu de l'ampleur du patrimoine pris en compte, que le seul jubé de la cathédrale de Limoges mérite une couverture systématique en 15 cartes $^{11}$ ? C'est alors que la collection s'ouvre plus largement à la sculpture étrangère. Cela nuance l'argument souvent avancé que, dès 1904, Enlart aurait voulu réduire le musée aux "monuments français ». L'explosion de la collection et son éclatement chronologique, comme la très large priorité données aux vues serrées des objets alors que les vues d'ensemble restent peu nombreuses, favorisent une histoire de l'art qui hausse le fragment au rang de fondement d'une modalité de la culture visuelle ; rendant possible des rapprochements, des combinaisons encore plus inédites que les galeries du musée qui réunissent déjà en un seul lieu l'essentiel de la sculpture répartie sur tout le territoire, voire une préfiguration d'un " musée imaginaire ${ }^{12}$ " portatif de la sculpture.

Après avoir présenté l'historique, le statut de cette extraordinaire collection et analysé son organisation en liaison avec la muséographie, nous tenterons d'en interpréter la dimension pédagogique et d'en proposer une lecture proprement historique, suivant le projet d'une sorte d'histoire en images d'Enlart et Roussel qui, d'ailleurs, avec le mouleur Pouzadoux se sont fait photographier dans les salles du musée (fig. 1), indiquant l'échelle (comme le fait si souvent Enlart dans ses travaux de terrain avec son épouse) et donnant également, comme dans une

8. «Le musée des Monuments français », http://www.culture.fr/Ressources/Moteur-Collections/ Autres-focus/Le-musee-des-Monuments-francais/(theme)/1

9. Le mot « moulages » est finalement rare aussi dans les publications : il n'apparaît pas dans le titre des albums de Frantz Marcou en 1897, ni sur les livres d'Enlart en 1911 et de Roussel en 1920 ; le catalogue de 1925-1928 omet le mot en couverture et le place entre parenthèses sur la page-titre ; il disparaît du catalogue de Denise Jalabert en 1943.

10. D'ailleurs le musée lui-même souffre d'un dédoublement des collections, d'abord limité à l'aile de Paris et étendu en 1889 à I'aile de Passy, sans qu'il soit envisageable de réorganiser l'ensemble, il y a donc double parcours historique ; puis en 1903, ce sont les galeries extérieures vitrées qui sont récupérées et dévolues à la sculpture étrangère sur l'aile de Paris et la sculpture avant le XIe siècle sur l'aile de Passy, complétée de la série de maquettes d'Anatole de Baudot.

11. Dans la première série, cartes nos 275 à 284, 340 (avec substitution d'Évreux ensuite); et on y revient en $661,685,693,753$.

12. La fascination de Malraux pour le fragment photographique est connue ; il écrit : « Le fragment, mis en valeur par sa présentation et par un éclairage choisi, permet une reproduction qui n'est pas un des plus humbles habitants du Musée imaginaire. », Les Voix du Silence, Guvres complètes, IV, Bibliothèque de la Pléiade, 2004, p. 213. Il usera des photos de moulages dans ses livres. 
mise en abyme, à saisir l'auteur en train d'écrire une histoire de la sculpture. Tout apparaît dans cette collection, même Madame Jalbert, la vendeuse de cartes postales, représentée avec son éventaire sous les arcs du porche nord de la cathédrale de Bourges (carte $\mathrm{n}^{\circ}$ 1176). Véritable musée miniature ou portatif ${ }^{13}$, cette collection fascinante possède encore une épaisseur historique, car certains des clichés pourraient être des prises de vue de Mieusement, et seront utilisés durant des décennies (même s'ils reproduisent une scénographie modifiée), au musée et sur tout le territoire par les Neurdein eux-mêmes, leurs successeurs, mais aussi des éditeurs locaux.

Figure 1:

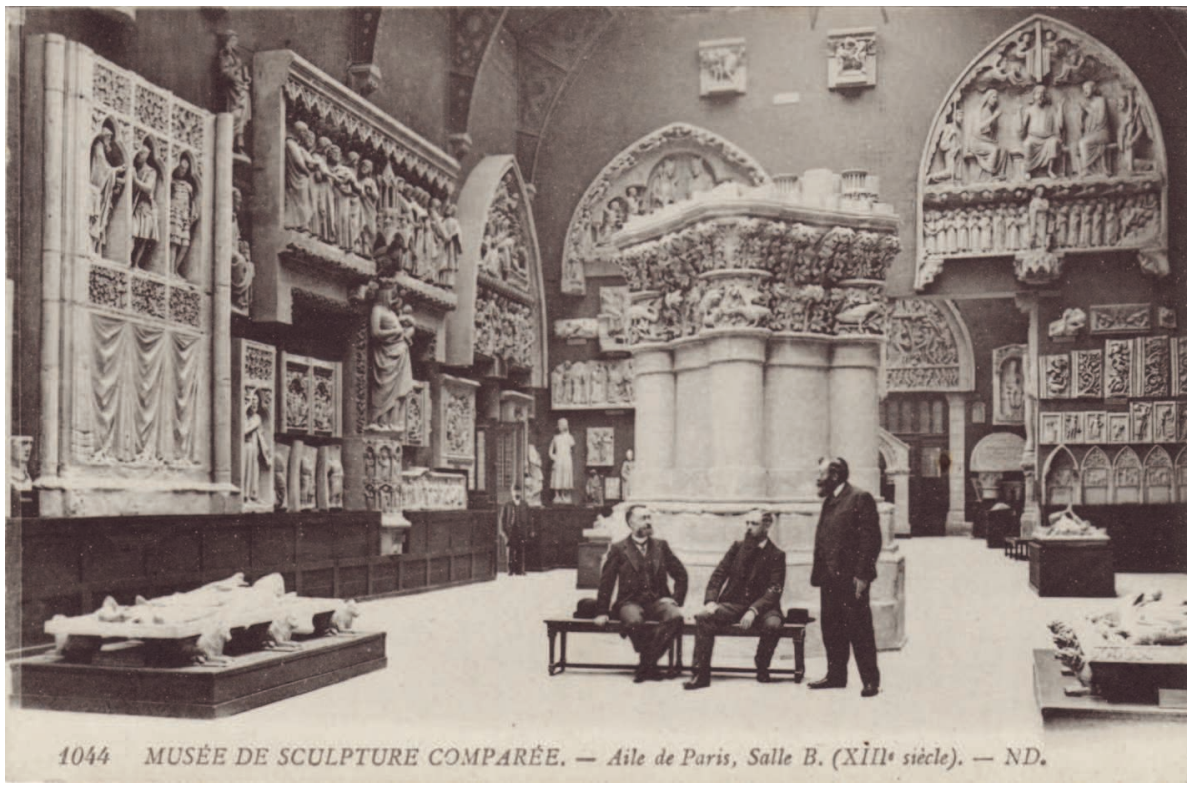

13. Ainsi que suggéré par Christine Lancestremère et Emmanuelle Polack dans « La photographie au musée de Sculpture comparée : médium de la diffusion de l'art français médiéval, instrument de comparaison intégré à la muséographie et apport documentaire à I'histoire de l'architecture », Revue de l'art, n 181, 2013-3, à propos, par exemple, des clichés de Paul Robert qui, selon l'Album du Musée de Sculpture comparée, publié sous la direction de P. Frantz Marcou, n'ont « d'autre prétention que de mettre sous les yeux du public un recueil d'images, qui fût comme un musée portatif de la sculpture française. » 


\section{Un point de vente au sein de la galerie d'exposition des moulages (aile de Paris)}

Le cahier des charges de la concession prévoit la possibilité de vendre des cartes postales, aux frais du concessionnaire, au musée de Sculpture comparée et au musée de Cluny mais aussi "dans tels lieux et telles conditions qu'il jugera profitables à la vulgarisation de la collection ${ }^{1}$ ». La carte postale ${ }^{\circ} 1176$ (voir fig. 34) représente le point de vente des photographies, catalogues et cartes postales du musée au sein de la galerie du musée de Sculpture comparée, appartenant aux éditeurs-photographes Neurdein. La femme présente sur l'image est très certainement Madame Jalbert, employée modèle, résidant au 35 rue Lecourbe à Paris, qui donne pleinement satisfaction à la maison Neurdein. L'opération commerciale est-elle rentable ? On peut le penser à la lecture d'une lettre de la Chambre syndicale de la photographie envoyée à la maison Neurdein en 1906, considérant que : « les musées, palais nationaux et édifices communaux n'ont pas vocation à vendre des cartes postales et des livres d'art ". La lettre dénonce une concurrence déloyale ${ }^{2}$ et demande que cesse immédiatement cette activité. Demande qui restera lettre morte. C'est la Grande Guerre qui mettra fin à la collaboration du musée de Sculpture comparée et de la maison Neurdein. Le traité de la concession, après avoir été renouvelé pour une période de cinq ans ${ }^{3}$, renouvelable de gré à gré, expire le 31 décembre 1915. Le 20 janvier 1916, la maison Neurdein, propriétaire de la collection des clichés de cartes postales du musée, souhaite alors vendre au musée le comptoir de vente et son stock. Le sort de l'employée méritante est évoqué ; les frères Neurdein recommandent chaleureusement la vendeuse Jalbert. Le matériel ${ }^{4}$ du comptoir de vente, travail d'ébénisterie en chêne de Hongrie, est proposé à l'administration des Beaux-Arts pour reprise.

D'une lettre de Camille Enlart, datée du 4 février 1916, on comprend le souhait du directeur de rétablir un dépôt de vente dans les galeries. « Le musée doit se rouvrir [sic] le $1^{\text {er }}$ avril et dès lors les artistes qui y travaillent comme les visiteurs qui y passent seraient déçus s'ils ne trouvaient plus à acheter comme par le passé dans nos galeries les catalogues et notices concernant le musée ; les cartes postales et les photographies des moulages qui le composent. [...] J'ai l'honneur de vous proposer cet achat ${ }^{5}$ "»

1. MAP 80/074/49 dossier 3 droits d'exploitation de la collection des Monuments historiques.

2. Ibidem.

3. Traité du 25 juillet 1898, 25 juillet 1903, 24 juillet 1908, 22 janvier 1913.

4. Il s'agit d'une table, de deux comptoirs avec support pour la présentation des cartes postales, d'un cadre, de quatre tourniquets en bois, d'un tourniquet en fer, de deux tourniquets en bois avec pieds en métal, d'un tabouret, le tout en bloc pour la somme de 1000 francs. MAP 80/074/49 dossier correspondance.

5. Archives des musées nationaux 5HH6 (2). 


\section{Historique de la collection des cartes postales}

Une lecture attentive des archives consignées dans le fonds du musée des Monuments français, et plus particulièrement, celles du fonds consacré au musée de Sculpture comparée (1882-1937), permet d'appréhender l'importance de la photographie au musée de Sculpture comparée. Dès les débats de la Souscommission ${ }^{14}$ des Monuments historiques, mise en ouvre en 1879 pour élaborer l'installation future du musée selon le vœu de Viollet-le-Duc, la photographie tient une place importante au cœur de ce projet, en participant de la volonté des acteurs de cette première muséographie de mettre en contexte les copies et d'étendre la diffusion des connaissances des arts de la période médiévale française. Le programme muséographique réalisé d'après un vaste choix des plus beaux monuments de la sculpture française met en regard des copies d'œuvres égyptiennes, grecques ou romaines.

Le 28 mai 1882, les premières salles du musée abritées dans l'aile Paris du Palais du Trocadéro ouvrent leurs portes au public. Le 18 avril 1883, Antonin Proust, président de la Sous-commission et directeur des Beaux-Arts, avance le nom du photographe Médéric Mieusement (1840-1905), connu d'Anatole de Baudot (1834-1915), pour avoir illustré son ouvrage sur la sculpture française publié en $1884^{15}$. Lors de la séance du 7 mars 1883, "M. le Président expose à la Commission l'intérêt qu'il y a à établir un bureau de vente de photographies au Musée. Mieusement est le premier qui ait demandé l'autorisation de vendre des photographies au musée. Il est décidé en conséquence qu'il sera invité à venir discuter les conditions auxquelles lui serait accordé le droit de vente et de tirage sur les clichés appartenant à l'administration ${ }^{16}$ ". Mieusement est ainsi autorisé par la sous-commission à vendre seul dans le musée, à un prix déterminé les photographies des moulages exposés. Paul Robert, son gendre et successeur en 1890 , continue sans autorisation à jouir de ce monopole. En avril 1898, à la mort de Paul Robert, sa veuve confie à J. Leroy fils les collections de photographies, ainsi que le modeste point de vente établi au sein des galeries du musée, déménagé le 17 mars 1899 comme le mentionnent les archives ${ }^{17}$.

La concession autorisant la vente des épreuves des clichés des Monuments historiques est mise en adjudication le jeudi 30 juin 1898 à trois heures au bureau des Monuments historiques au 3, rue de Valois à Paris. Le règlement stipule que " nul ne sera admis à concourir s'il ne justifie pas de la qualité de français et s'il n'est pas éditeur-photographe ayant un atelier de tirage à Paris ${ }^{18}$ ". Les frères Neurdein, Étienne (1833-1918) et Louis-Antonin (1846-1914), photographes, éditeurs et imprimeurs, remplissant ces deux qualités, obtiennent la concession par traité le 25 juillet $1898^{19}$. On peut aussi souligner leur lien de filiation avec Mieusement ; ils sont ses neveux.

L'usage de la collection des clichés des monuments historiques confiés à la garde du musée de Sculpture comparée est affermé à cette entreprise privée tenue de livrer à un prix modéré des épreuves à toute personne qui en fait la demande. En contrepartie, le concessionnaire se charge des travaux d'entretien de la dite collection de clichés, et ce, à hauteur de 1500 francs annuellement, s’astreignant également à verser à l'État une somme avoisinant les 200 francs. La

14. Créée le 3 novembre 1879, la sous-commission se compose de MM. Antonin Proust, directeur des Beaux-Arts, Jules Quicherat (1799-1884), directeur de l'École des chartes, Émile Boeswillwald (1815-1896), Juste Lisch (1828-1910), Victor Rupricht-Robert (1820-1887), inspecteurs généraux, Anațole de Baudot (1834-1915), inspecteur général des édifices diocésains, Castagnary, conseiller d'État, Alexandre Denuelle, Gustave Dreyfus (1837-1914), Gautier, contrôleur des travaux, Adolphe Victor Geoffroy-Dechaume (1816-1892), statuaire, Robert de Lasteyrie (1849-1929), professeur de l'École des chartes, Poulin, directeur des Bâtiments civils, Edmond Du Sommerard (1817-1885), Steinheil (1818-1885), peintre, Treteau, conseiller d'État. 15. Voir Anne de Mondenard, Photographier l'architecture 1851-1920. Collection du Musée des Monuments français, Paris, 1994. Catalogue de l'exposition au musée national des Monuments français du 20 mars au 20 juin 1994 et au musée des Beaux-arts de Marseille, Palais de Longchamp, du $1^{\text {er }}$ juillet au $1^{\text {er }}$ septembre 1994.

16. Archives du musée de Sculpture comparée, cote CAPA/MSC carton 0.

17. J. Leroy retire du musée du Trocadéro les objets suivants lui appartenant à savoir : une table, une vitrine, deux albums, deux chemises d'épreuves H. 0,30; L. 0,40 m et sept chemises d'épreuves H. 0,21; L. 0,27 m. Archives de la Médiathèque du patrimoine, cote MAP cote 80/074/01, dossier 1.4

18. MAP cote $80 / 074 / 49$.

19. MAP cote $80 / 074 / 49$, dossier 4 , correspondances. 
mise en adjudication de la concession de la vente des clichés photographiques des Monuments historiques et de la collection du musée de Sculpture comparée obtenue par la maison Neurdein semble avoir suscité des ambitions rivales. Les éditeurs et les imprimeurs sont nombreux à signaler leur intérêt en formulant des demandes d'autorisation de photographier les œuvres du musée sur papier timbré à l'adresse du directeur de l'institution muséale. Ce sont, par exemple, l'éditeur-photographe Lévy et ses fils, 25, rue Louis-le-Grand et dont l'atelier se situe au 44, rue Letellier à Paris, qui sollicite cette permission le 24 juin $1897^{20}$ et le $1^{\text {er }}$ juillet $1901^{21}$ ou bien encore L. Hudson, 5, rue Ordener à Paris, spécialiste de reproductions artistiques et de cartes postales illustrées ${ }^{22}$ (fig. 2-5).

Figure 2

Lettre à en-tête Lévy et ses fils Archives CAPA/ MSC 14 / chemise 1897 c) Cité de l'architecture et du patrimoine

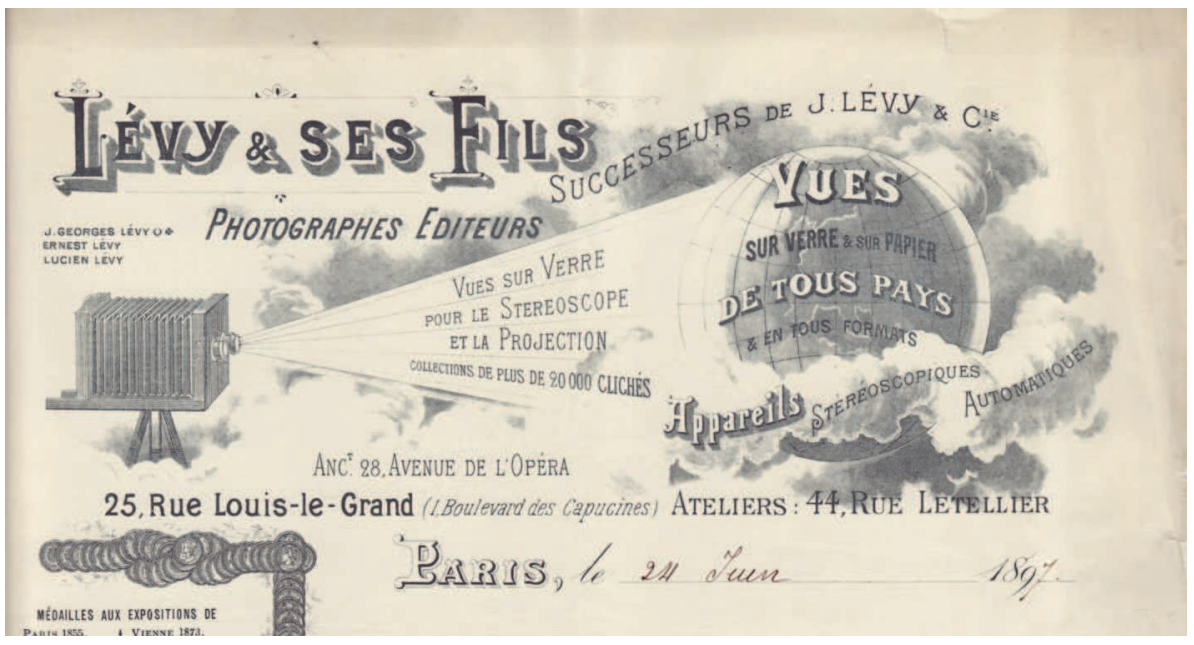

Figure 3

Lettre à en-tête Lévy et ses fils Archives CAPA/MSC 14/ chemise 1901 Cite de larchitecture et du patrimoine
-Editions. Photographiques. MÉDAILLE D'OR S'Exposition Universelle de Paris 4900
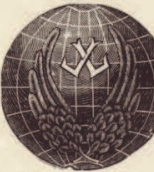

Marque L. L.

-o-

DREsSE TELLËGaPAPHQUE NEGATIF-PARIS

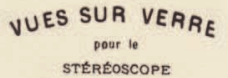
VUES SUR VERRE STERÉOSCOPE STÉRÉOSCOPE Cartes Postales illustrées dous PAYs. EN TOUS FORMATS

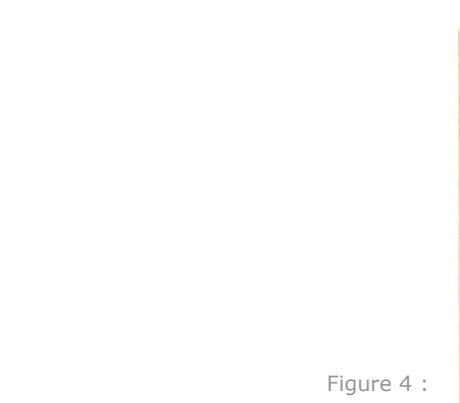

Lettre à en-tête Neurdein Archives CAPA/ MSC14 / chemise 1910 c) Cité de l'architecture et du patrimoine

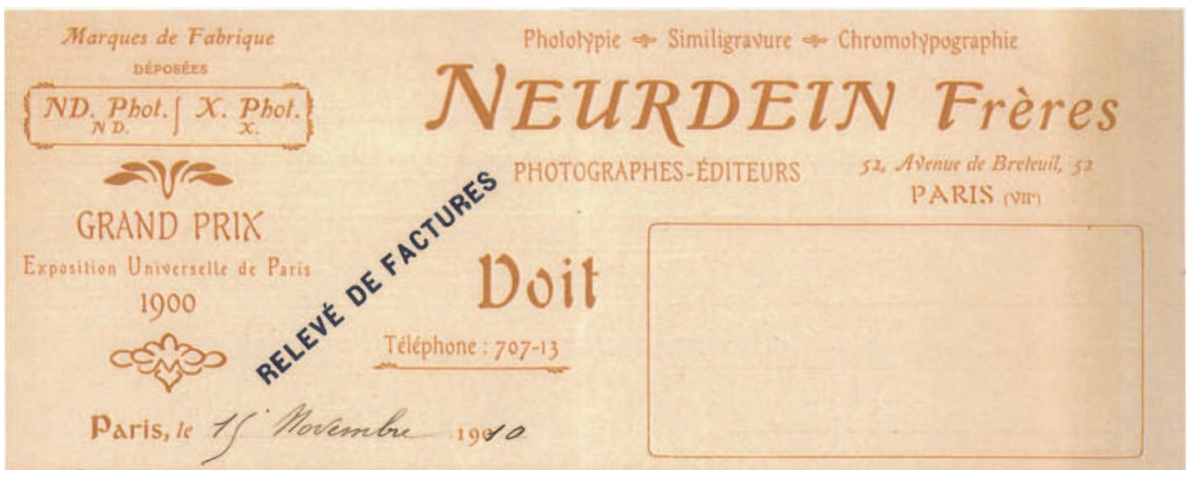


Figure 5

Lettre à en-tête L. Hudson Archives CAPA/ MSC 14 / chemise 1911 c) Cité de l'architecture et du patrimoine

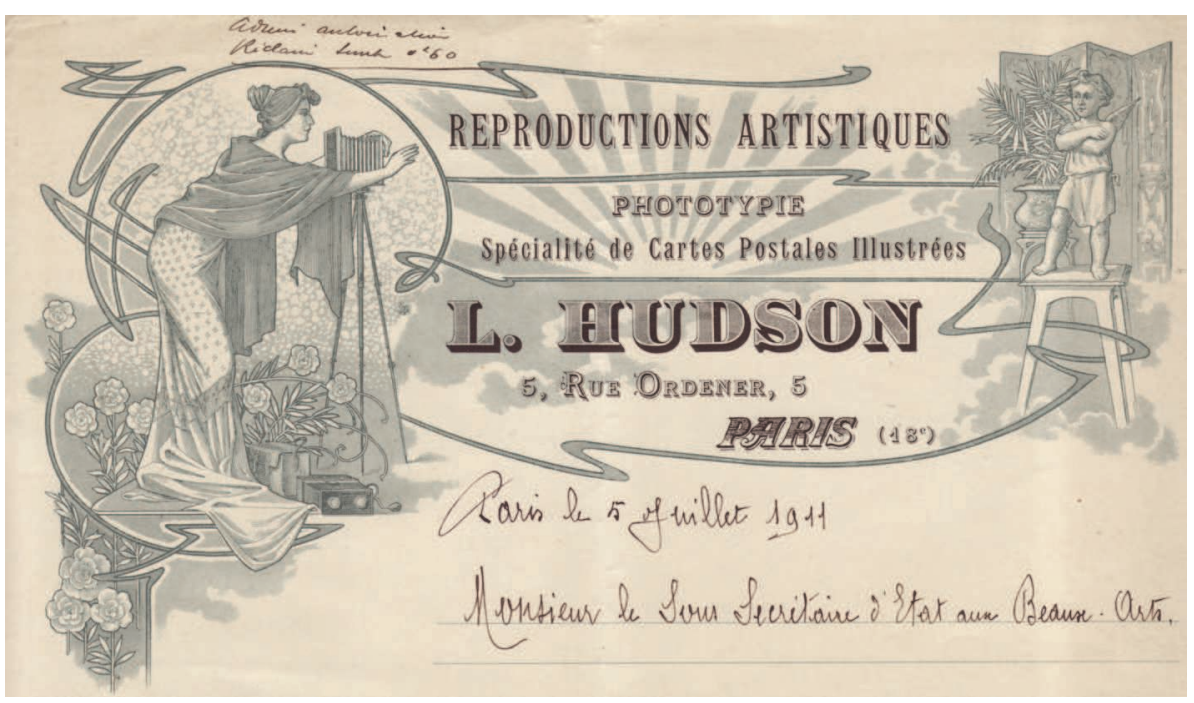

Léditeur, de premier plan, Ernest Le Deley, dont le siège social se trouve au 127, boulevard de Sébastopol dans le $2^{\mathrm{e}}$ arrondissement de Paris, propose de son côté des cartes stéréoscopiques et une série d'excellente qualité numérotée, mais non poursuivie au-delà de quelques dizaines ${ }^{23}$. Datées de 1900 , on observe sur celles-ci, le monogramme stylisé ELD ainsi qu'un grainage plus fin, gage d'une phototypie de grande qualitée ${ }^{4}$ (fig. 6-8).
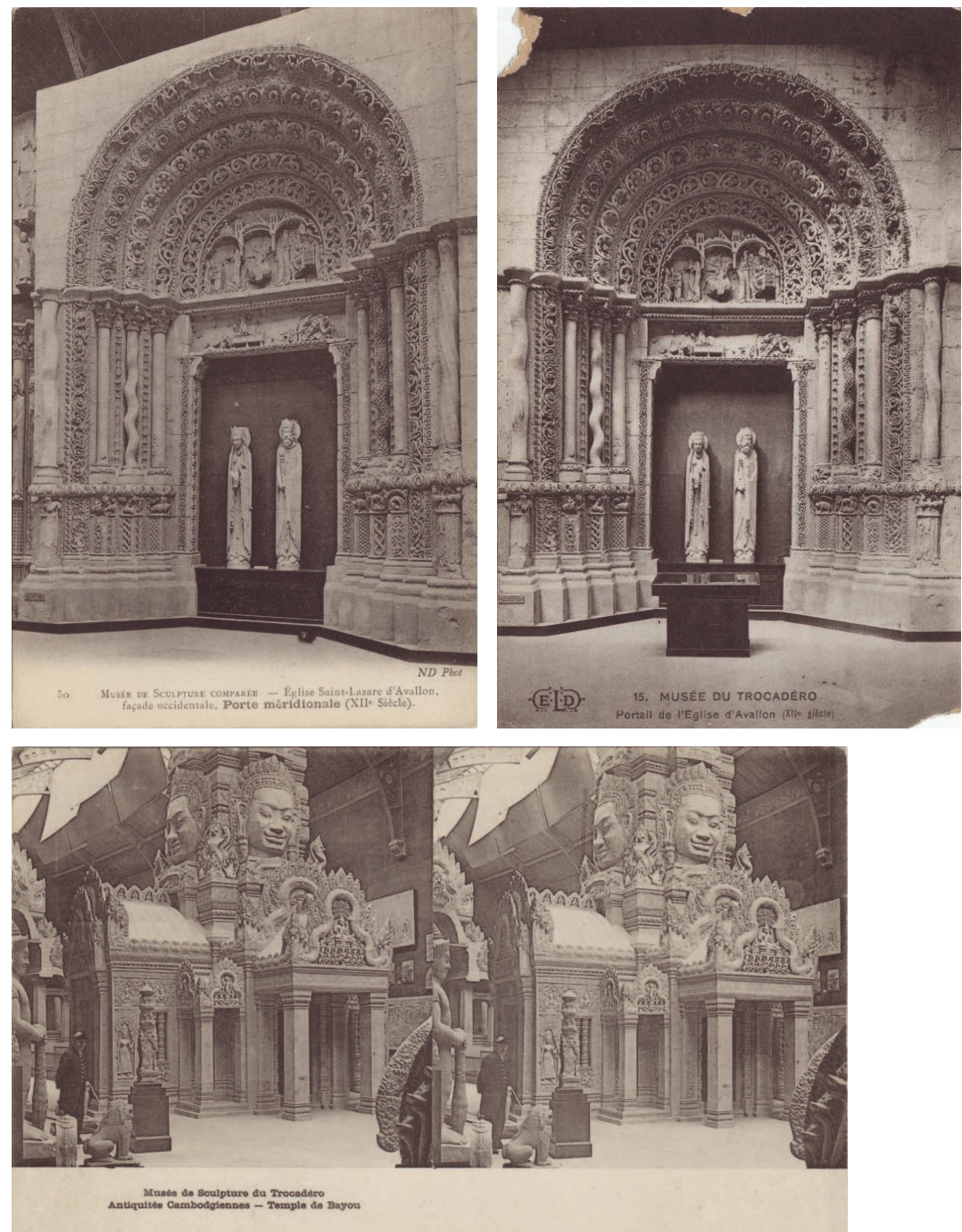

23. Sous les titres « Musée de sculpture du Trocadéro » et « Musée du Trocadéro ».

24. Les auteurs tiennent à remercier Françoise Ploye, restauratrice de photographies anciennes et contemporaines pour cette observation. 
La Maison des frères Neurdein fondée en 1864, établie tout d'abord au 28 boulevard de Sébastopol, avant de rejoindre la très chic adresse du 52, avenue de Breteuil s'est rendue célèbre par la vente de portraits historiques, puis de photographies réalisées à la Tour Eiffel, opération commerciale dont le succès fut prodigieux selon les termes d'Étienne Neurdein. Les Salons de 1887 à 1908 ont été également à l'origine de leur fortune ainsi que les Expositions universelles de Paris. La qualité de leur travail est récompensée en 1878 par une médaille d'argent, en 1889 une médaille d'or et en 1900 par un Grand prix.

Lors des années 1902, 1903 et $1904^{25}$, les demandes d'autorisation de photographier les moulages font florès de la part des amateurs et des visiteurs du musée. De ce constat, Camille Enlart (1862-1927) " amoureux des images ", a-t-il souhaité, dès sa prise de direction du musée de Sculpture comparée en 1903, initier une importante campagne d'édition de cartes postales du musée ? En l'état actuel de nos connaissances, nous ne pouvons le confirmer. Cependant, il est certain que des prises de vues existantes, un concessionnaire désigné, on pouvait aisément passer à la fabrication de cartes postales. Cette série éditée très certainement à la fin de l'année $1904^{26}$ par les frères Neurdein pour le musée de Sculpture comparée est composée tout d'abord d'une première série de 486 cartes recensées dans leur catalogue des Moulages dont il a été exécuté des reproductions en carte postale, sous-titré Des collections et sujets édités dans le format carte postale par Neurdein Frères, photographes, éditeurs et imprimeurs, dont l'édition nous semble avoir été livrée vers l'année $1905^{27}$.

Les cartes postales reproduites en noir et en blanc, selon un procédé mécanique de phototypie, sont signées dans un premier temps "ND Phot »; dès les années 1910, le sigle évolua avec le ND inscrit dans la boucle du P (Neurdein et Cie), pour finir pour les retirages en simple "ND ", lors de la fusion "L. Lévy et Neurdein ${ }^{28}$; enfin on trouve " ND PHOT. " avec dos vert qui correspond à la phase après le rachat par l'imprimerie Crété (1921). Les légendes figurent au recto de la carte postale précisant le nom de l'institution, le nom de l'édifice original, du fragment représenté, sans aucune mention de son état de moulage, mais elles ne sont pas toujours fiables. Elles ne sont pas sans poser quelques difficultés aux éditeurs mais, plus encore, au directeur du musée de Sculpture comparée, qui fait part de son mécontentement quant aux " mentions erronées des indications de monuments, de départements et même de localités ${ }^{29}$ ". En 1905, des corrections seront apportées aux légendes erronées qu'elles soient liées aux fausses indications de département, de localité ou aux dénominations des édifices originaux ${ }^{30}$. De même, les numérotations ne sont pas sans problème : l'Autel gallo-romain $\mathrm{n}^{\circ} 13$ réapparait sous le $\mathrm{n}^{\circ} 1097$; la vue de la salle avec le Puits de Moïse est numérotée 176, comme une semelle de poutre d'Anvers. Un même cliché des moulages est utilisé par les Neurdein pour le musée, mais aussi pour la ville où se trouve l'original : par exemple la carte ${ }^{\circ} 498$ présente les élus du tympan de la cathédrale de Bourges; le même cliché numéroté 175 est légendé : "Bourges. Cathédrale Saint-Étienne... les élus ». Le même cliché de la Visitation est légendé " Musée de sculpture comparée... " ( $\left.{ }^{\circ} 300\right)$ et "Troyes. Église SaintJean... " (fig. 9, 10), les sculptures du chœur de Chartres dans le contexte du musée sont légendées comme "Cathédrale de Chartres " sans mention qu'il s'agit des moulages (fig. 11). Cas inverse, la carte $\mathrm{n}^{\circ} 224$ qui correspond au tombeau de Charles IV au Mans, dans la version de la carte conservée par les archives du Musée ne représente pas le moulage, mais l'original dans sa niche (fig. 12, 13).

25. Archives CAPA/MSC 14 chemise 1902.

26. Le verso divisé des premiers numéros des cartes postales laisse penser qu'elles ont été mises en fabrication après le 4 janvier 1904.

27. Catalogue consultable à la Bibliothèque nationale de France, sur le site Richelieu, sous la cote AD1822 (110).

28. La suite de l'histoire de la collection reste à écrire : ainsi se sont encore des photos ND (identiques aux CP) qui accompagnent la plaquette de Jules Roussel en 1920, alors que le catalogue de 1929 précise que les clichés sont «Lévy-Neurdein » et offre quelques nouvelles prises de vue. Plus tard, une série de cartes, « Musée de sculpture comparée » comporte les numéros ND, mais est éditée par Moutet, « Aux musées d'Europe » : par exemple, ND. 271 La Mort de Ligier-Richier.

29. MAP 80/074/49 Dossier correspondance.

30. Idem, ibidem. 

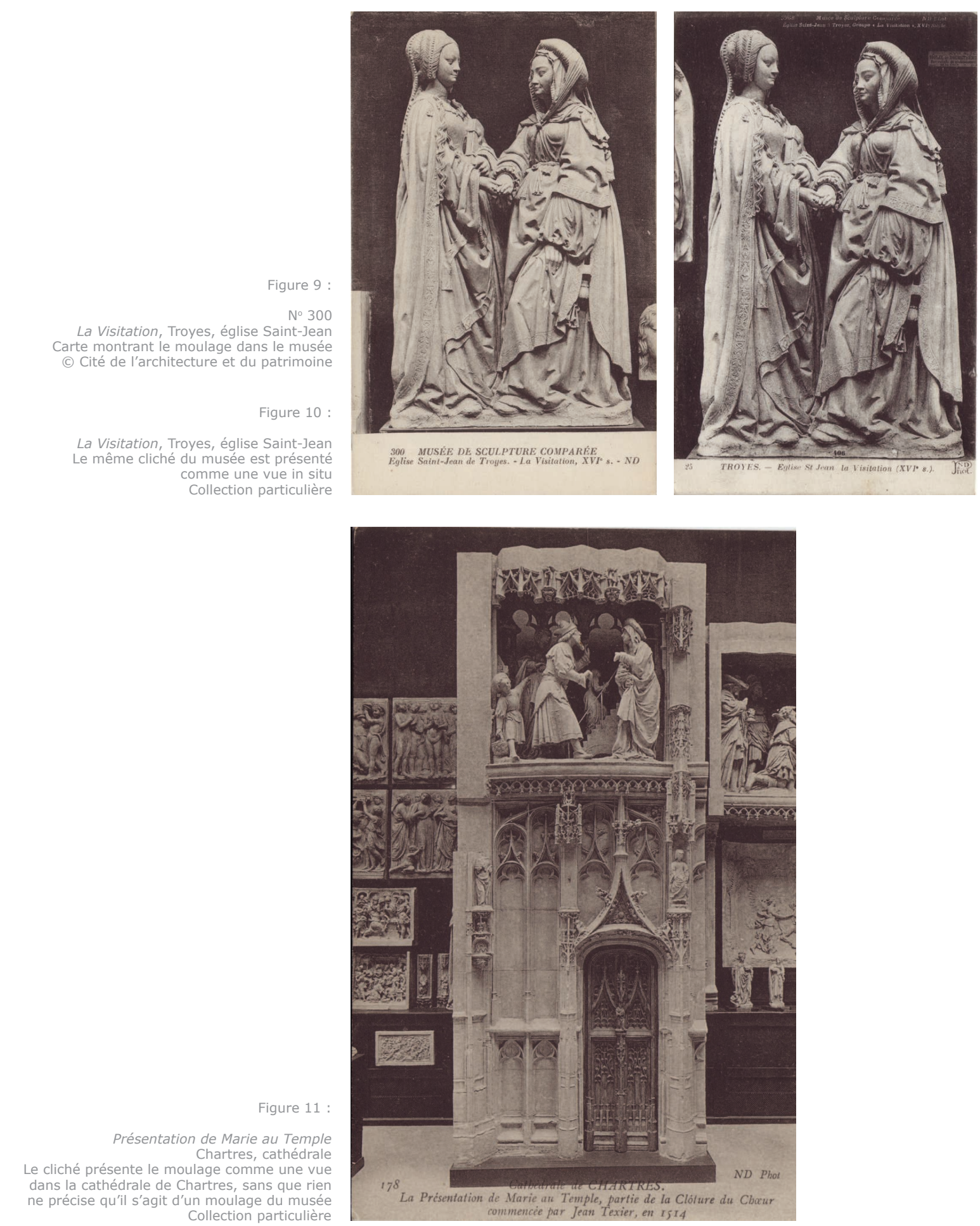

Figure 12

№ 224. Tombeau de Charles IV d'Anjou Le Mans, cathédrale. La carte représente la vue du tombeau dans la cathédrale, mais le donne comme un moulage du musée (c) Cité de l'architecture et du patrimoine

Figure 13

№ 224. Tombeau de Charles IV d'Anjou Le Mans, cathédrale. Une nouvelle carte numérotée 224 montre cette fois le moulage
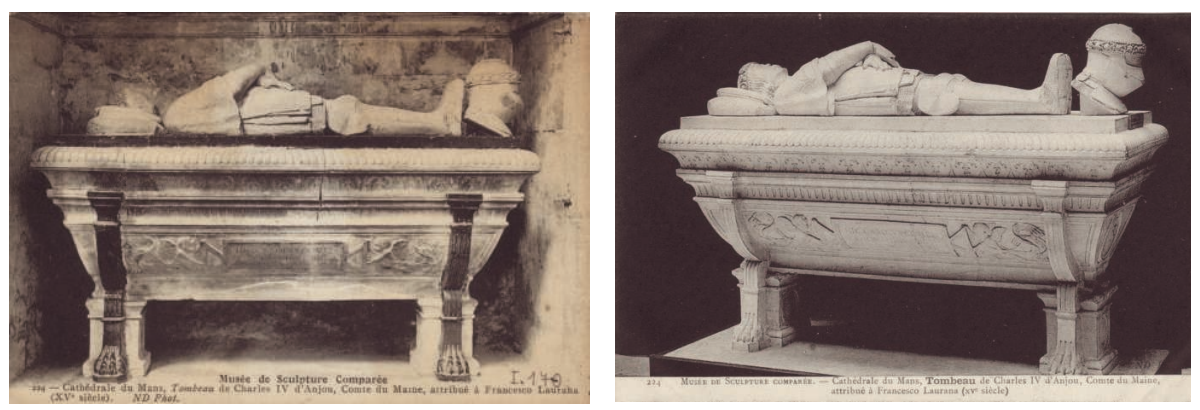


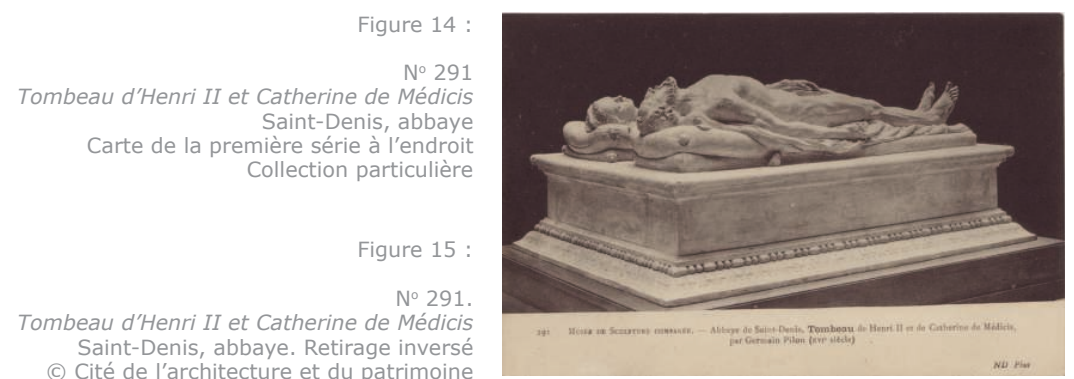

Mais il existe aussi une carte du moulage. Au retirage parfois, des clichés sont inversés, tel le tombeau d'Henri II (no 291) (fig. 14, 15).

Il convient encore de signaler une forme de commercialisation qui introduit bien des confusions et montre comment aux yeux des éditeurs le moulage tend à équivaloir l'original. Les frères Neurdein vendaient des clichés des moulages du musée de Sculpture comparée à des éditeurs locaux qui imprimaient leur marque et vendaient les cartes comme des vues des monuments de localités où ils exerçaient : ainsi le papetier Herbout d'Amiens ou l'éditeur Toulot d'Auxerre vendent des vues des moulages de la cathédrale de leur ville. Les acheteurs de cartes n'étaient guère plus scrupuleux, une carte envoyée de Chartres par un visiteur n'est autre qu'une vue d'un moulage de la tête de sainte dans la cathédrale $\left(\mathrm{n}^{\circ} 115\right)$.

\section{La collection de cartes postales : un témoin archéologique de la scénographie du musée de Sculpture comparée}

Les premières cartes offrent donc un parcours historique cohérent, qui n'est autre que celui qui présidait déjà à la présentation des salles du musée des années 1890 repris par les publications de Paul-Franz $\mathrm{Marcou}^{31}$ : Époque gallo-romaine$\mathrm{XII}^{\mathrm{e}}$, XIII', $\mathrm{XIV}^{\mathrm{e}}-\mathrm{XV}^{\mathrm{e}}$, XVII ${ }^{\mathrm{e}}-\mathrm{XIX}{ }^{\mathrm{e}}$ siècles (fig. 16).

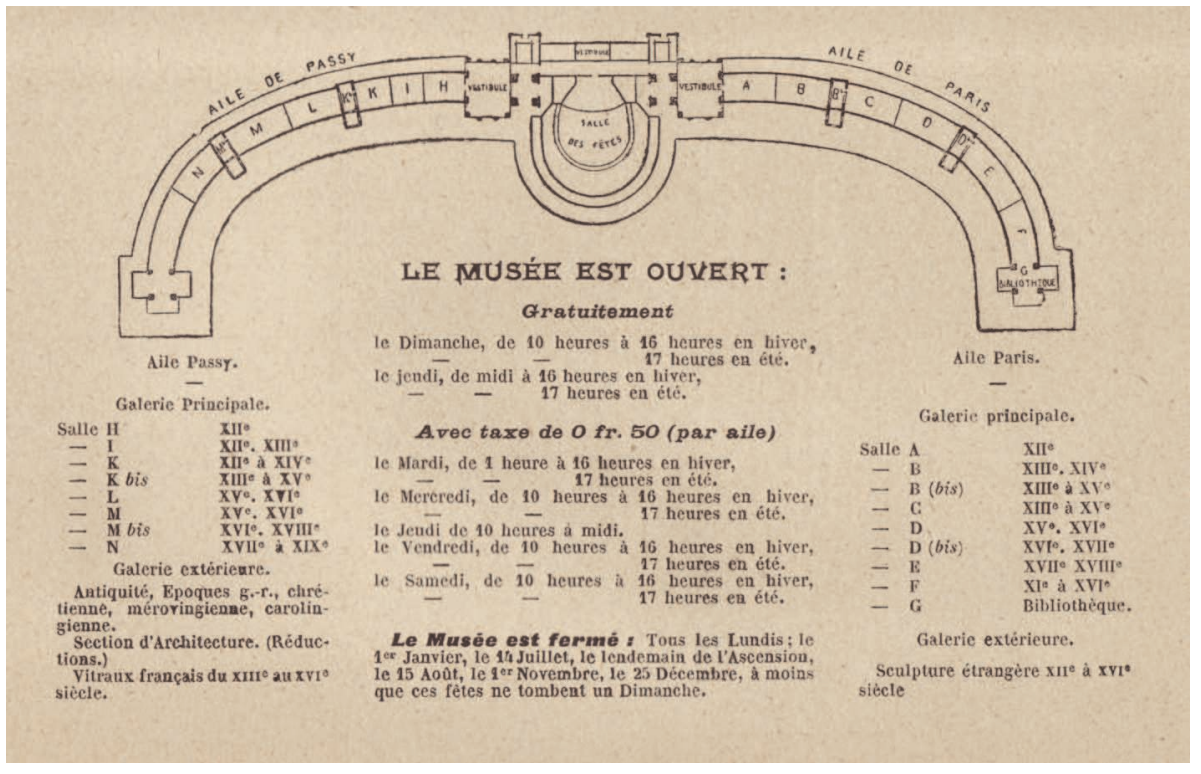

31. Album du Musée de sculpture comparée (palais du Trocadéro).... sous la direction de Paul Frantz Marcou, Paris, s. d., $1^{\text {re }}$ série, Époque gallo-romaine-XII ${ }^{\mathrm{e}}$ siècle ; $2^{\mathrm{e}}$ série, XIII ${ }^{\mathrm{e}}$ siècle ; 3e série, $X I V^{e}$ et $X V^{e}$ siècles. 
Les six premiers numéros renseignent le musée Indo-Chinois ${ }^{32}$, implanté sur environ un cinquième de la surface totale de l'aile de Passy du Palais du Trocadéro (fig. 17, 18). Les numéros suivants reprennent la démonstration comparative de Viollet-le-Duc qui souhaitait des moulages des arts égyptiens, assyriens et de la période hiératique grecque ${ }^{33}$ en regard des œuvres des $\mathrm{XI}^{\mathrm{e}}$ et $\mathrm{XII}^{\mathrm{e}}$ siècles français (fig. 19, 20). Paul Deschamps, le successeur d'Enlart, y fait encore allusion : «On pouvait y voir, à côté de moulages de sculpture égyptienne du Musée de Boulaq, de sculptures grecques et romaines des Musées de Munich, de Londres, de Naples et du Louvre, de grandes pièces de sculpture monumentale choisies parmi les plus imposantes et les plus belles de l'art français des époques romanes, gothique et de la Renaissance ${ }^{34}$ ". Suivent quelques moulages de monuments gallo-romains (fig. 21). Commencent ensuite les grandes périodes auxquelles sont dévolues les reconstitutions les plus prestigieuses, le style roman du $\mathrm{n}^{\circ} 16$ (Notre-Dame du Port) au n 75 (Saint-Rémi de Reims), en passant par Moissac (fig. 22, 23), SaintGilles du Gard, Vézelay ou Autun, puis le style gothique, du n 76 (Notre-Dame de Reims) au $\mathrm{n}^{\circ} 179$ (Puits de Moïse), regroupant les plus célèbres cathédrales (Amiens, Paris, fig. 24), Chartres, Laon, mais aussi Naumbourg) (fig. 25, 26). Le catalogue des frères Neurdein classe les cartes par siècle depuis le XII siècle. Cela permet d'évaluer les proportions qui, là encore, ne répondent pas aux idées reçues : 70 cartes (180 à 251) couvrent le XVe siècle et s'ouvrent plus largement à l'étranger, Florence (fig. 27) ou Amsterdam ; le XVI e siècle est illustré par 114 cartes (252 à 366) (fig. 28), soit plus que les XIII et XIV e siècles! Il y a donc un indéniable intérêt pour cette période qu'on associe moins que le Moyen Âge à ce musée. Le $\mathrm{XVII}^{\mathrm{e}}$ siècle a droit à une série de 86 cartes, le XVIII ${ }^{\mathrm{e}}$ seulement 27 (fig. 29); enfin le XIXe siècle qui clôt le cycle avec les Rude de l'Arc de Triomphe, n'est présenté qu'en 5 cartes. Symboliquement la carte $\mathrm{n}^{\circ} 486$ (fig. 30), qui clôt la première série, représente l'éphèbe de Rude, le dernier moulage que l'on voyait, salle N, avant de pénétrer dans la section cambodgienne.

Figure 17

1. Entrée de sanctuaire. Cambodge (c) Cité de l'architecture et du patrimoine

Figure 18

2. Temple de Bayon. Cambodge Collection particulière
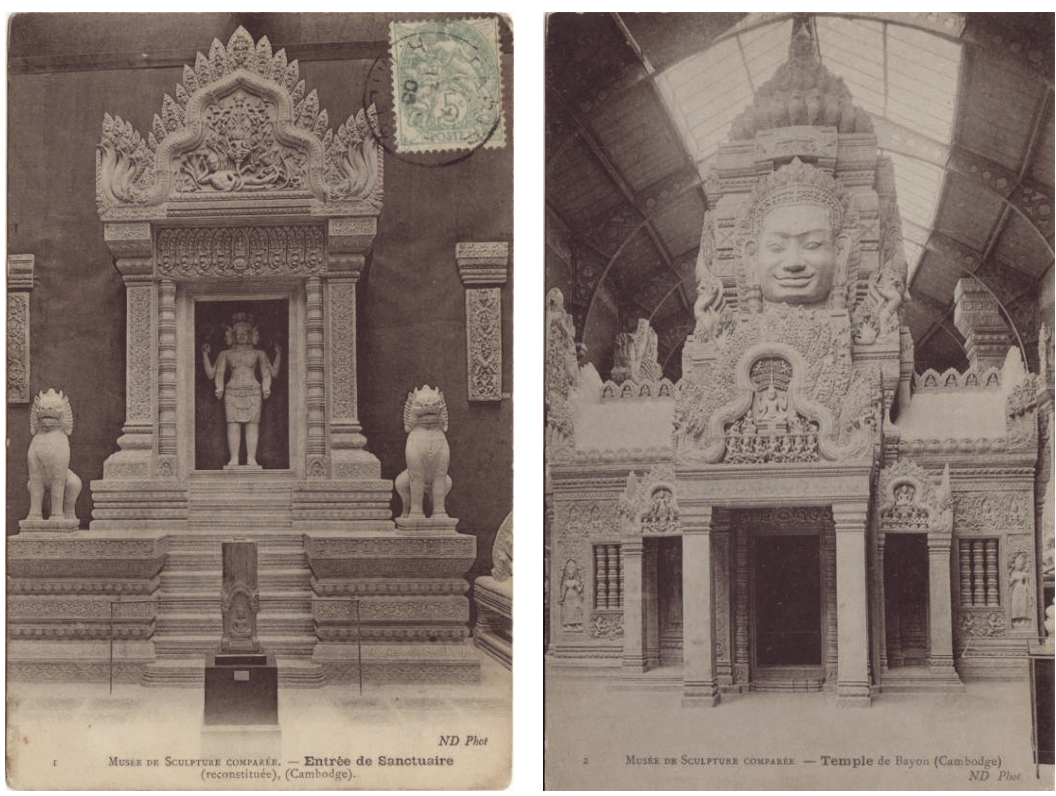

32. Louis Delaporte, officier de Marine, visite le site d'Angkor en 1866. Au cours d'une seconde mission, toujours à Angkor, en 1873, il rapporte en France de nombreux moulages, exécutés par les premiers pionniers français qui se rendirent au Cambodge au XIX siècle mais également, des schémas, des plans et des œuvres originales. La collection trouve, tout d'abord, refuge au Château de Compiègne, avant d'être installée dans une salle de l'aile de Passy du Palais du Trocadéro en 1882. Louis Delaporte est nommé conservateur des collections khmères du Palais du Trocadéro qui, plus tard, devient le musée Indochinois du Trocadéro, et qu'il dirige jusqu'en 1924. Les moulages se retrouvent à I'Exposition Coloniale Internationale de Vincennes en 1931, après un passage partiel par celle de Marseille en 1906 et 1922. À partir de 1935, lors des travaux de la transformation du Palais du Trocadéro, se pose le problème de l'entreposage de la collection du musée Indochinois. Les moulages sont déposés et stockés par le service des Bâtiments civils dans une ancienne usine appartenant à M. Appert au 9 rue Valiton à Clichy. Les œuvres originales, quant à elles, viennent enrichir les collections du musée Guimet.

33. Les moulages d'antiques seront installés dans la galerie latérale de l'aile de Passy, avant d'être retirés des collections en 1933, pour être y être conservés à I'Institut de l'art.

34. Paul Deschamps, Le Musée de Sculpture Comparée. Extrait du Congrès de Paris 1934, t. 1. Version dactylographiée, archives du Musée, CAPA/MSC3, p. 2, 3. 
3. Cheik-el-Beled. IV dynastie Statue trouvée dans un puits funéraire de la nécropole de Memphis

Figure 20

11. Cariatide provenant du Temple de l'Érechthéion. (c) Cité de l'architecture et du patrimoine
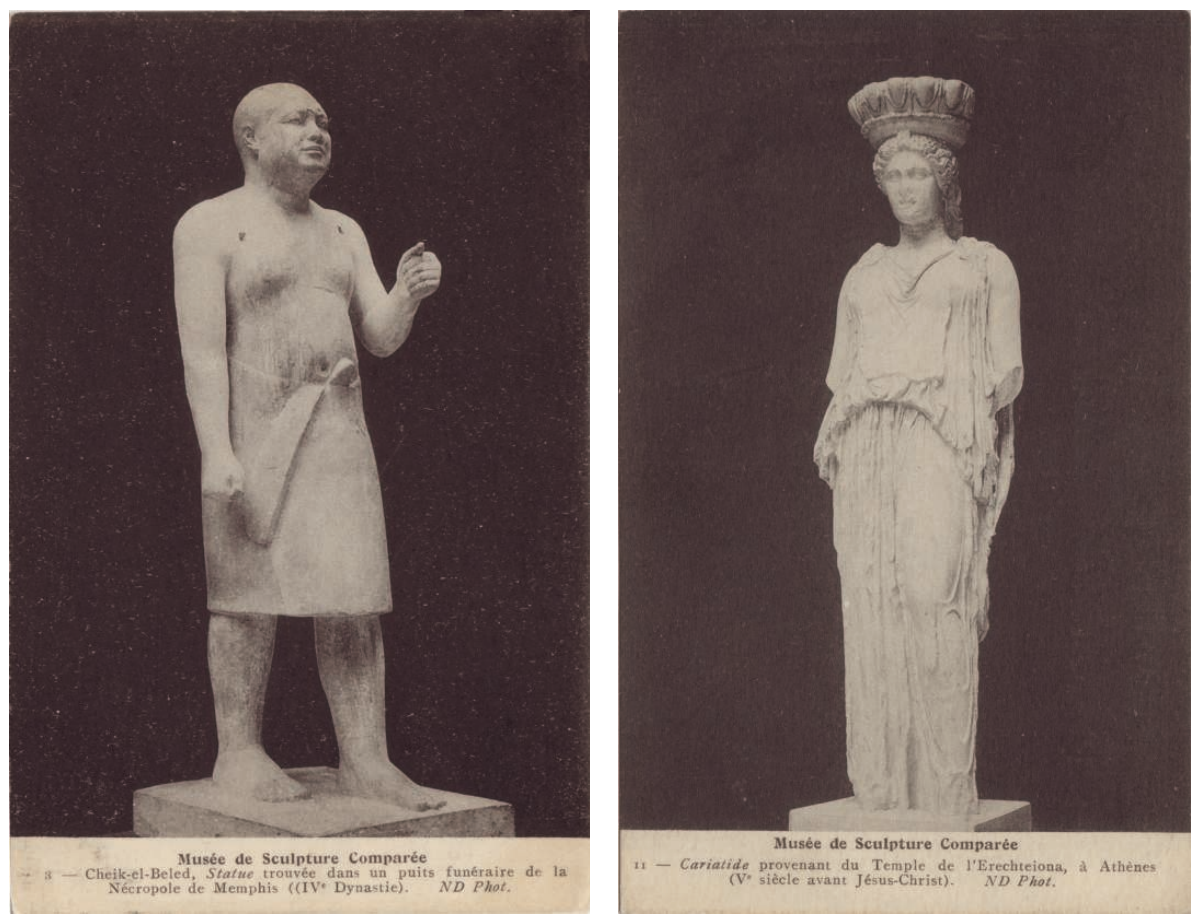

15. Un Sanglier. III siècle (c) Cité de l'architecture et du patrimoine

Figure 22 :

25. Portail latéral du porche occidental XII siècle Moissac église Saint-Pierre (c) Cité de l'architecture et du patrimoine

Figure 23 :

25. Version de la carte précédente détourée (c) Cité de l'architecture et du patrimoine
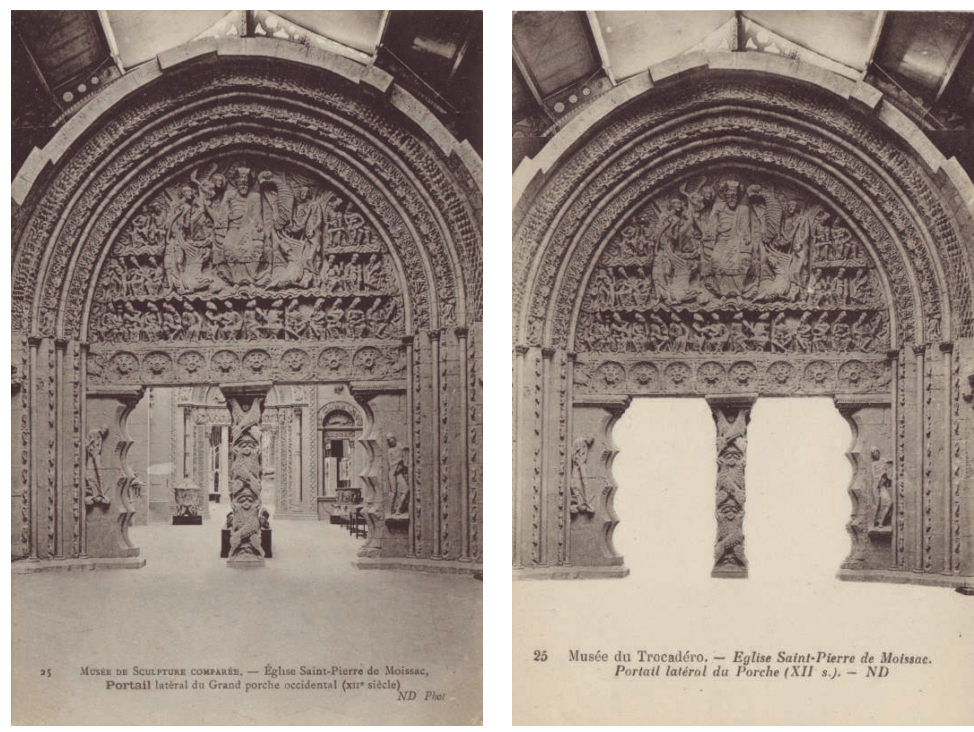
Figure 24

93. Tympan du portail de la Vierge Paris, cathédrale Notre-Dame. La carte porte la cote du moulage dans le catalogue d'Enlart et Roussel qui permet de le situer dans la salle $C$ (c) Cite de l'architecture et du patrimoine

Figure 25:

146. Statue de la comtesse Baba. XIII siècle Naumbourg, cathedrale

Figure 26

147. Statue du comte Eckart. XIII ${ }^{\mathrm{e}}$ siècle (C) Cité de l'architecture et du patrimoine

Figure 27

226. La Vierge et I'Enfant. Michel-Ange Bruges, église Notre-Dame

Figure 28:

307. Tombeau de Louis de Brézé XVIe siècle, Rouen, cathédrale (c) Cité de I'architecture et du patrimoine

Figure 29

479. Fontaine de Neptune. XVIU e siecle, Fantaine Place Stanislas c) Cité de l'architecture et du patrimoine

Figure 30 :

486. Rude, Éphèbe de l'Arc de triomphe de /'Etoile. XIXe siècle
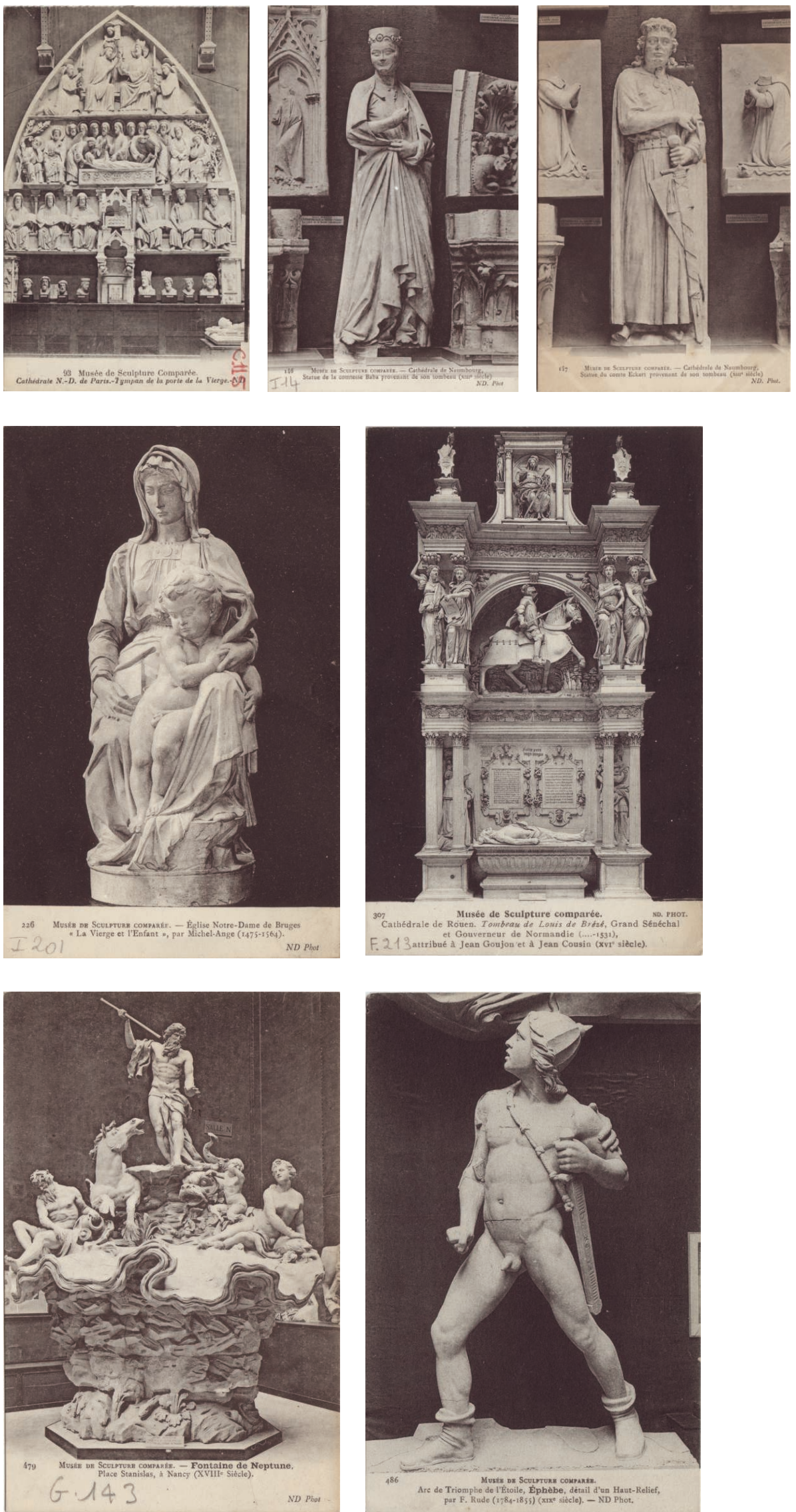
On ne doit pas surinterpréter cette série de cartes, car il y manque des monuments majeurs ! Et les proportions sont trompeuses, puisque Paul Deschamps écrit encore en 1934 que "La Renaissance est insuffisamment représentée " $^{35}$ ". C'est pourquoi les frères Neurdein s'empressèrent de compléter la collection, en abandonnant le parti-pris chronologique et en complétant la documentation sur des monuments traités succinctement dans la première série. La seconde série qui se développa au moins jusqu'au $\mathrm{n}^{\circ} 1606$, introduit des arts nouveaux, les maquettes d'Anatole de Baudot et, pour finir, quelques vitraux ; elle permet aussi de percevoir des changements scénographiques apportés par Enlart dans les années 1903-1905. Ainsi une pièce importante comme le Puits de Moïse est déplacée : les cartes $n^{\text {os }} 176^{36}$ et 252 (fig. 31-33) la montrent au centre de la salle des $\mathrm{XV}^{\mathrm{e}}$ et XVI $\mathrm{XI}^{\mathrm{e}}$ siècles (D), la carte $\mathrm{n}^{\circ} 1176$ atteste son installation dans la salle B' derrière le portail de la cathédrale de Bourges (fig. 34). Deux versions de la carte 177 (fig. 35, 36) montrent ce changement de cadre.

Figure 31 :

176. Galerie orientale salle des XVe et XVIe siècles C Cité de l'architecture et du patrimoine

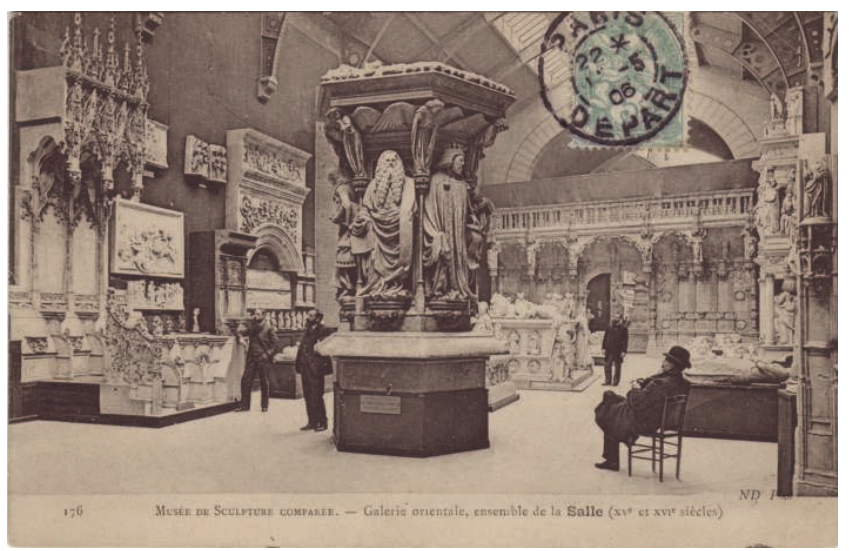

Figure 32 :

176. Galerie orientale salle des XVe et XVI siècles c Cité de l'architecture et du patrimoine

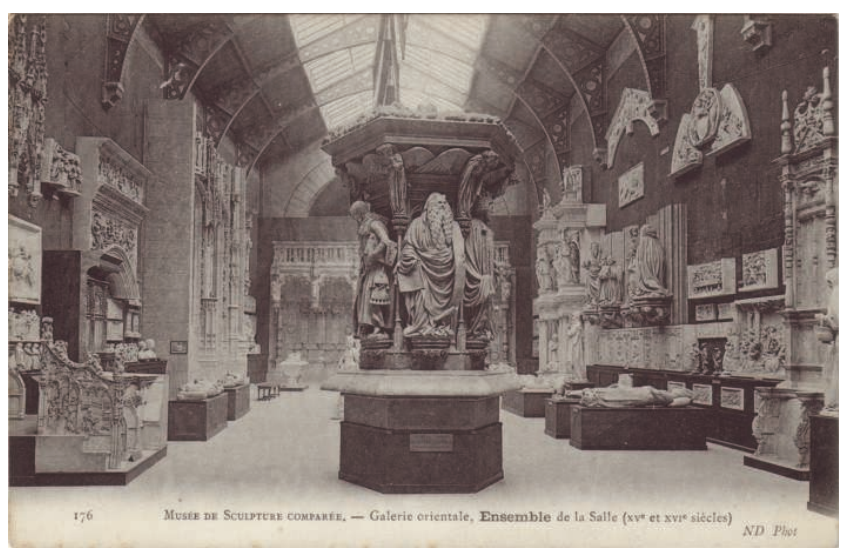

Figure 33

252. Une salle de l'aile occidentale [sic] XVIe siècle. Exemple du manque de rigueur dans les légendes

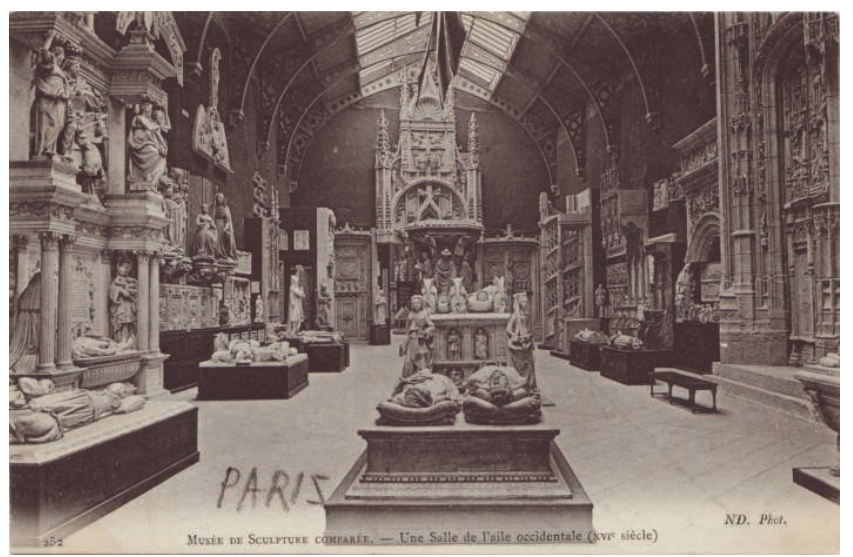

35. P. Deschamps, op. cit. note 34, p. 7.

36. Précisons qu'il existe deux versions de cette carte de même époque «ND Phot », une vue, prise dans l'axe de la galerie, avec le Puits de Moïse au centre, I'autre prise de biais avec quatre personnages, qui sera retirée en «ND». 
Figure 34 :

1176. portail. Bourges, cathédrale La petite salle $D^{\prime}$ accueille outre le Puits de Moïse
le comptoir de Madame Jalbert (c) Cité de l'architecture et du patrimoine
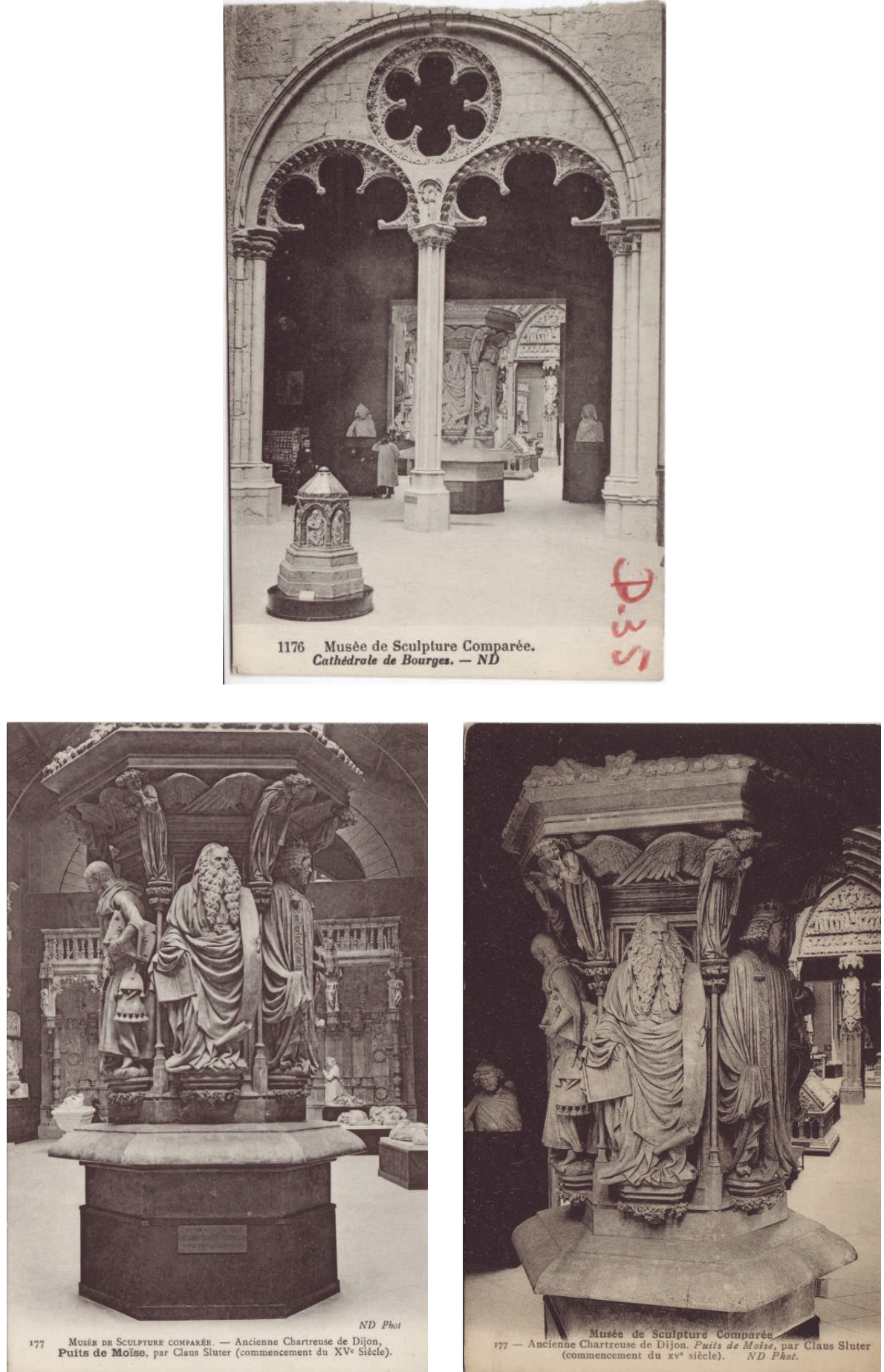

En réunissant les cartes de la première série qui présentent des moulages situés à proximité du Puits de Moïse, on peut arriver à une reconstitution minutieuse de la scénographie d'un secteur qui a été modifié par Enlart. Il n'est pas sans signification de mettre en perspective le Puits avec les tombeaux d'Henri II et de François II, de l'entourer des fragments de Saint-Maclou de Rouen, de stalles de Saint-Bertrand de Comminges et d'Amiens, mais évidemment aussi de les associer aux pleurants du tombeau de Jean sans Peur. Toute la salle se retrouve globalement entre les cartes $\mathrm{n}^{\circ} 180$ (début des Pleurants) et $\mathrm{n}^{\circ} 327$ (couronnement à SaintPierre de Caen) ; ce qui est énorme par rapport aux autres salles. Des ensembles aux numérotations suivies s'y perçoivent, dont la continuité n'est justifiée que par la disposition des moulages : ainsi les 224, tombeau de Charles IV d'Anjou dans l'angle et 225, porte de la sacristie de Bourges. Le décor de Gaillon (290) voisine avec le tombeau d'Henri II $(291,292)$, les stalles d'Amiens $(293,294) \ldots$ 
En revanche seules la carte 176 donne une vue d'ensemble, que peut compléter la carte 293 qui donne le contexte de la stalle d'Amiens. Or cette scénographie est modifiée entre 1904 et 1910, date du Catalogue général illustré qui s'ouvre sur une vue de la salle des XV et $\mathrm{XVI}^{\mathrm{e}}$ siècles, avec les auteurs, mais où le Puits a laissé la place à la Croix de Saint-Cirques ( ${ }^{\circ}$ 823). Les cartes 176 témoignent d'une organisation d'avant 1910 et sont donc à utiliser avec circonspection.

Il convient de noter la rareté de ces vues d'ensemble ; n’auraient-elles pas été plus synthétiques et séduisante pour les visiteurs ? Or, en dehors de quelques portails complets comme Saint-Gilles du Gard ou Vézelay, de quelques fontaines, les vues de salles restent peu fréquentes ${ }^{37}$. Cela est cohérent avec les deux tendances majeures qui se discernent dans les choix des vues qui composent la collection de cartes postales : le goût du fragment - en relation avec la base même du principe du moulage - et l'exhaustivité.

Les cartes postales offrent donc des cadrages serrés sur les objets pris dans leur ensemble, puis décomposés dans leurs différents détails décoratifs ou motifs iconographiques : ainsi les Pleurants sont présentés par quatre, puis individuellement ; de même chaque allégorie du tombeau de François II. Ce principe, apparu dans la première série, est repris pour certains œuvres et amplifié dans la seconde : les tombeaux de Marguerite de Bourbon (1438-1483), Philibert-le-Beau (1480-1504) et de Marguerite d'Autriche (1480-1530) à Brou dont chaque Sybille ou saint est individualisé, ou encore la stalle d'Amiens qui été présentée globalement (293) et avec son dais (294) et qui fait l'objet d'une vingtaine de photos supplémentaires (1234-1244, 1256-1263, 1273, 1281). Il est significatif que des cartes (expérimentales ?) aient été produites qui présentent un découpage du motif : l'image de cette stalle d'Amiens (293) entourée des moulages de Gaillon, des Pleurants et des bas-reliefs de l'hôtel du Bourgthéroulde représentant le Camp du drap d'or, devait paraître confuse, aussi a-t-on tiré une carte $^{38}$ où elle est découpée (assez maladroitement) et apparaît sur fond blanc pour isoler l'objet (fig. 37, 38) ; le même type de découpe se retrouve sur des cartes du Beau Dieu d'Amiens (fig. 39, 40). Dans la seconde partie de la collection, la démultiplication permet de reproduire des séries énormes de chapiteaux, de vierges à l'enfant, jetant les bases d'études typologiques.
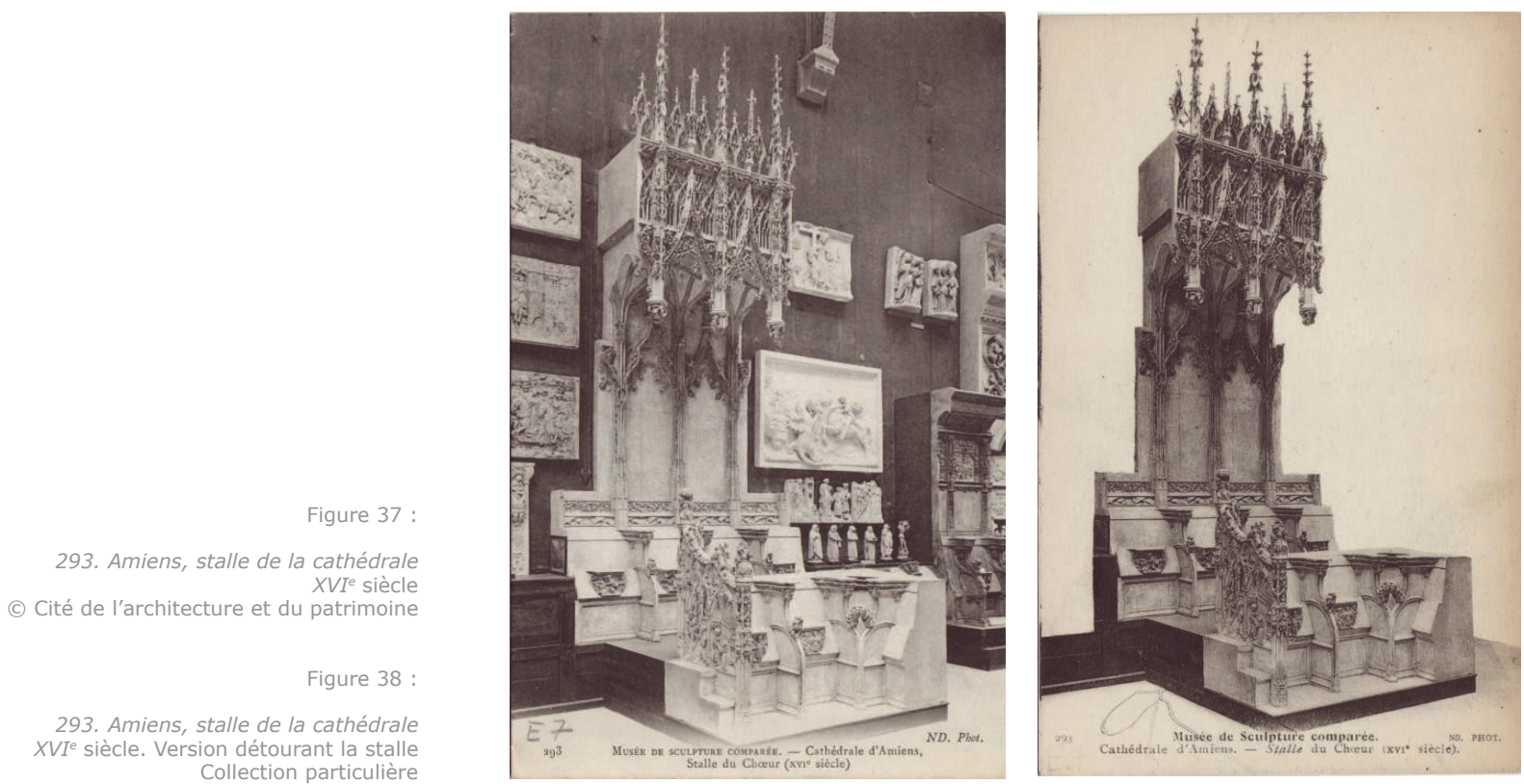

37. Hormis le temple d'Angkor (2), Vézelay (45), la salle $x V^{e}-x V I^{e}$ siècles(176) décrite ici, la chapelle d'Avioth (233), la salle M avec I'hôtel de Berny à Toulouse (341) et la salle du XVII siècle de l'aile de Passy (367), la première série comporte assez peu de vue des salles ; la seconde série en propose encore moins : I'aile de Paris, salle B, XIII ${ }^{\mathrm{e}}$ siècle (1044), le portail de Bordeaux (1506)

38. Carte « ND. PHOT. » de la période Imprimerie Crété, donc des années 1920. 

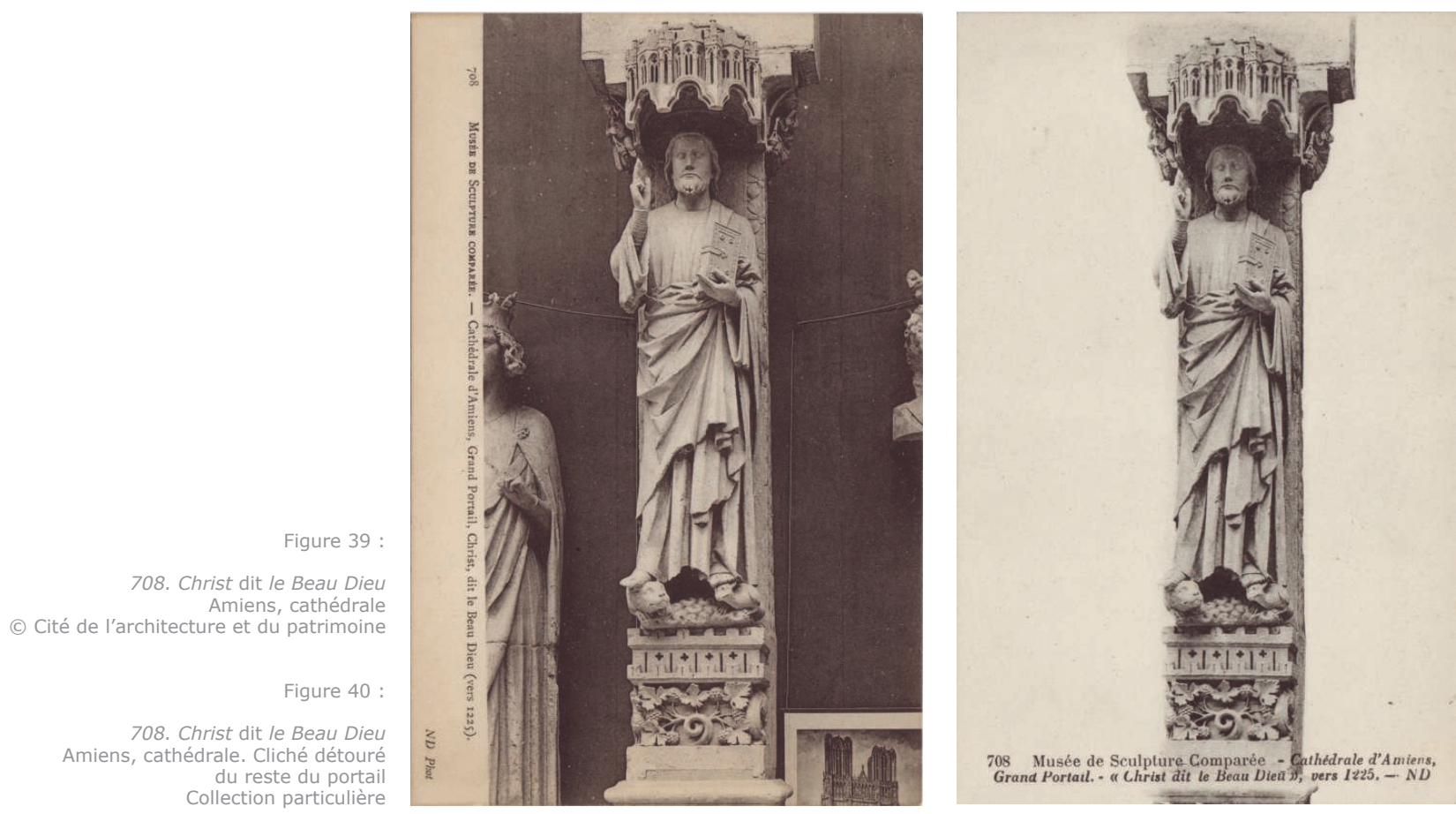

Y eut-il recherche d'exhaustivité ? Bien sûr, cette collection de cartes postales ne remplace pas les photos des moulages régulièrement réalisées, mais elle semble néanmoins atteindre une dimension systématique ; celles des archives du musée comportent, écrit au crayon, la cote du moulage dans les salles, dans la scénographie d'Enlart ${ }^{39}$, reprise dans le catalogue général. Ce grand nombre de cartes pouvait ainsi servir à la gestion pratique des collections. Deschamps rappelle qu'en 1936, le musée conservait 6945 moulages, c'est bien sûr beaucoup plus que les 1600 cartes postales crées : néanmoins, si l'on se souvient qu'un moulage peut faire l'objet de plusieurs cartes, une carte peut représenter plusieurs objets, surtout en matière de sculpture ornementale, cela donne une proportion d'objets reproduits impressionnante, au moins un tiers. Il y a donc bien un projet qui, comme le moulage, ressort à la diffusion d'une histoire de l'art en images libérée des contraintes de l'original.

\section{Modalités d'une histoire visuelle de la sculpture}

Un colloque avait émis en 1999 - cent-cinquantenaire de la mort de Violletle-Duc - l'audacieuse hypothèse qu'au musée de Sculpture comparée s'était jouée la "naissance de l'histoire de l'art moderne $~^{40}$ : Roland Recht, en particulier, y avait montré la fidélité au projet scientifique de Viollet-le-Duc dont la construction théorique tendait à donner un statut central au Moyen Âge français, en s'appuyant sur une vision évolutionniste et une méthode comparative empruntée aux sciences naturelles. Sans revenir sur cette dimension, il convient de montrer comme la collection de cartes postales poursuit à sa manière le projet tout en l'infléchissant par le processus même de diffusion, de reproduction mécanique des reproductions. Car il faut se souvenir qu'une des données fondamentales du musée était d'offrir des moulages grandeur, à l'échelle $1 / 1$, d'où l'impression grandiose des tympans romans, des escaliers, des tombeaux : on rivalise bien avec les colonnes grandeur réelle du Parthénon de la cour des études de l'École des Beaux-Arts.

39. Selon aussi le Catalogue général illustré de 1910 dont Enlart et Roussel précisent bien qu'il s'agit d'une édition entièrement refondue, avec les phototypies des frères Neurdein frères. C'est évidemment l'état le plus proche de la collection de cartes postales.

40. Le Musée de Sculpture Comparée : naissance de l'histoire de l'art moderne, Paris, Monum/ Éditions du Patrimoine, 2001. 
Roland Recht insistait surtout sur la proximité entre moulage et photographie ${ }^{41}$, rappelant que le musée était tout autant consacré à la photo qu'aux moulages, celle-ci étant le complément de l'objet pour le contextualiser, mais aussi une source documentaire largement exploitée. Il précisait encore que le moulage comme la photo permet le cadrage, le détourage, de ce fait une analyse formaliste. La carte postale perpétue, en la facilitant, cette pratique, car sur 1600 cartes il n'est guère qu'une vingtaine de vues de salles qui montrent l'agencement du musée, les autres privilégiant le cadrage serré sur un moulage, voire un détail, telles les miséricordes des stalles d'Amiens ; certains ensembles sont aussi détourés, le « beau Christ » ou les stalles d'Amiens. La fragmentation de la composition devient un principe de lecture qui favorise aussi l'approche stylistique.

En 1600 cartes, Enlart et les frères Neurdein écrivent donc une histoire visuelle qui partage la fonction du catalogue, en portant l'accent sur le visuel, comme le tentaient les albums de Marcou dans les années 1890. Ce qu'écrivent Enlart et Roussel à propos de leur catalogue est vérifiable dans leur série monumentale de cartes postales, elle "constitue donc une véritable histoire de la sculpture française et un aperçu de la sculpture étrangère ${ }^{42}$. "

Sans être le substitut du catalogue raisonné, commencé en 1892 par Louis Courajod et Paul-Frantz Marcou et jamais achevét ${ }^{43}$, la collection de cartes postales poursuit les ambitions majeures du musée initial, voire de Violletle-Duc : innombrables sont les fragments « décoratifs » qu'elle offre à l'amateur ou à l'artisan, répondant au troisième principe fondateur défini par Viollet-le-Duc en 1879, à côté de la comparaison entre " différentes époques et civilisations » et de la comparaison interne à la sculpture française "par écoles ", l'" application de la sculpture suivant le système d'architecture employé " ${ }^{44}$, c'est-à-dire la "sculpture d'ornement ou décorative ${ }^{45}$ " qui suit les mêmes évolutions : Viollet-le-Duc réclamait cet enseignement pour les artistes.

On peut dire que les 486 premières cartes, comportant quelques références égyptiennes et grecques, des monuments gallo-romains maintiennent le principe comparatif premier, alors qu'il est rompu, dans les salles, par le déplacement des antiques dans les galeries latérales. Cependant si la part belle est réservée aux grandes époques romanes et gothiques et à la Renaissance et aux chefs-d'œuvre de Goujon et de Bouchardon, l'amplitude chronologique, du gallo-romain à Rude et Carpeaux, et les monuments peu connus retenus construisent un ensemble d'une étonnante variété, une culture sculpturale de référence. L'usage de ces cartes, d'abord collectionnées ${ }^{46}$ et utilisées comme références par les artistes ${ }^{47}$, traduit un projet scientifique clair, comme l'atteste la primauté donnée aux fragments et aux détails sur les vues pittoresques des salles. Le comparatisme est rendu encore plus aisé par la facilité des combinaisons de ces photos détachées. Véritable instrument scientifique, la collection s'est chargée de la même valeur que l'original, selon l'argumentation de Courajod et Marcou qui écrivaient :

"Si des notices parfois assez étendues ont été consacrées à de simples moulages, c'est qu'on a pensé qu'il n'y avait pas, en matière de sculpture, de distinction à faire entre l'œuvre elle-même et sa reproduction ; c'est aussi que, pour l'histoire de l'art, un moulage, qui ne peut être suspect d'interprétation, a toute la valeur scientifique d'un original et mérite, par

41. Le Musée de Sculpture Comparée : naissance de l'histoire de l'art moderne, op. cit. note 40, p. $52-53$

42. Camille Enlart et Jules Roussel, Musée de Sculpture comparée du Trocadéro. Catalogue général, Paris, Henri Laurens, 3 fascicules réunis, 1925-1928, s. p. Les 96 planches du catalogue sont des clichés Lévy-Neurdein.

43. Musée de sculpture comparée (moulages). Palais du Trocadéro. Catalogue raisonné... XIVe et $X V^{e}$ siècles, Imprimerie Nationale, 1892 ; avec les clichés de Paul Robert.

44. Viollet-le-Duc, Musée de Sculpture comparée appartenant aux divers Centres d'art et aux diverses époques, Imprimerie Bossier, 1879, p. 3.

45. Idem, ibidem, p. 8.

46. Comme le prouvent le fait que l'essentiel des cartes qui se vendent aujourd'hui sur le marché de l'occasion sont vierges, et que I'on trouve des ensembles de cartes dans les collections ou les ateliers.

47. Ainsi le sculpteur Réal del Sarte, qui lui-même diffusa très largement son œuvre et la propagande johannique en cartes postales, possédait une sélection de cartes du musée de Sculpture comparée, en particulier une série de vierges à l'enfant. 
suite, d'être examiné avec le même intérêt. Aussi, n'a-t-on pas craint de publier ici, comme on l'aurait fait en présence des originaux s'ils eussent été réunis dans un musée, le texte même des pièces, comptes, mémoires, marchés, mandements, de payement, etc ${ }^{48}$."

Si nous avons consacré tant de signes à ces simples cartes postales, ce n'est pas que copies de copies, elles vaillent les originaux, mais parce que nous estimons qu'il s'y inscrit une histoire visuelle qui mérite une exploration plus systématique, riche de potentielles réinterprétations sur l'écriture de l'histoire de la sculpture. C'est aussi que nous souhaitions démontrer la nécessité de ne pas y voir une simple documentation transparente (ne serait-ce qu'en raison aussi des erreurs qu'elles véhiculent), mais le matériau même d'une histoire et que ces cartes comme collection et comme objets de collection ont, non seulement acquis le droit à une patrimonialisation légitime, mais à une étude pour elles-mêmes.

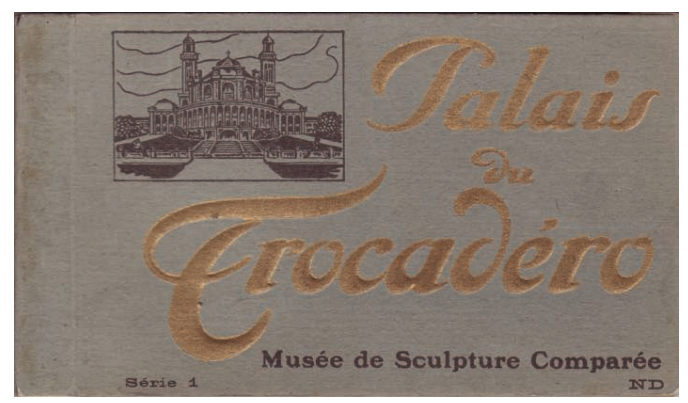

\section{Les auteurs}

De formation historienne, Emmanuelle Polack est en charge des archives du musée des Monuments français au sein de la Cité de l'architecture et du Patrimoine. Elle a assuré en 2010 le commissariat de l'exposition "Rose Valland sur le front de l'art » au Centre de la Résistance et de la Déportation à Lyon. Elle est aujourd'hui chercheur(e) associée sur le programme "Marché de l'art sous l'Occupation » au sein de l'Institut national d'histoire de l'art.

Dominique Jarrassé est professeur d'histoire de l'art contemporain à l'université de Bordeaux Montaigne et à l'École du Louvre. Il a fait ses études à Nancy et sa thèse à Paris 4 sur L'architecture thermale en France entre 1800 et 1850 . Ses travaux concernent l'art et l'architecture du XIX ${ }^{e}$ et de la première moitié du XXe siècle, particulièrement l'architecture des synagogues et l'art juif. Il a publié divers ouvrages comme L'Âge d'or des synagogues (1991), Rodin (1993), Une histoire des synagogues françaises. Entre Occident et Orient (1997), L'art des jardins parisiens (2002), Existe-t-il un art juif ? (2006)... Il aborde aussi des questions touchant à l'historiographie de ces domaines et aux relations de l'art et de l'anthropologie. Il a intégré l'Équipe de recherche de l'École du Louvre en octobre 2012. 